\title{
TWO-DIMENSIONAL RIEMANN PROBLEM FOR A SINGLE CONSERVATION LAW
}

\author{
TONG ZHANG AND YUXI ZHENG
}

\begin{abstract}
The entropy solutions to the partial differential equation
$(\partial / \partial t) u(t, x, y)+(\partial / \partial x) f(u(t, x, y))+(\partial / \partial y) g(u(t, x, y))=0$,

with initial data constant in each quadrant of the $(x, y)$ plane, have been constructed and are piecewise smooth under the condition $f^{\prime \prime}(u) \neq 0, g^{\prime \prime}(u) \neq 0$, $\left(f^{\prime \prime}(u) / g^{\prime \prime}(u)\right)^{\prime} \neq 0$. This problem generalizes to several space dimensions the important Riemann problem for equations in one-space dimension. Although existence and uniqueness of solutions are well known, little is known about the qualitative behavior of solutions. It is this with which we are concerned here.
\end{abstract}

\section{INTRODUCTION}

The Riemann problem [1, 2] has been a key to the development of the theory of one-dimensional conservation laws. For multidimensional conservation laws, similar Riemann-type problems may be considered. Their study is interesting and valuable not only in theoretical aspects but also in fluid mechanics; for example, the diffraction of a planar shock along a compressive corner is a special case. However, the problem in several space dimensions is much more complicated. As a beginning towards a general theory, the present paper deals with the problem for a single conservation law. Although existence and uniqueness of solutions to the single conservation law with general initial data has been obtained earlier, it is useful to solve the Riemann problem in an exact manner both for understanding the qualitative behavior of solutions and for applications to other problems.

The two-dimensional Riemann problem for a single conservation law is

$$
\begin{array}{r}
\partial u / \partial t+\partial f(u) / \partial x+\partial g(u) / \partial y=0 \\
u(0, x, y)= \begin{cases}u_{1}, & x>0, y>0 \\
u_{2}, & x<0, y>0 \\
u_{3}, & x<0, y<0 \\
u_{4}, & x>0, y<0\end{cases}
\end{array}
$$

Received by the editors September 16, 1986 and, in revised form, February 5, 1987.

1980 Mathematics Subject Classification (1985 Revision). Primary 35L65, 35L67; Secondary $65 \mathrm{M} 99$.

The contents of this paper were presented to the Workship on Nonlinear Hyperbolic Waves held at the Mathematical Sciences Research Institute at Berkeley, California, from May 25-27, 1987. 
where $f$ and $g \in C^{3}(-\infty,+\infty)$. Many papers deal with the problem [4-7]. Wagner [4] constructed the entropy solutions in the sense of Kruzkov [3] under the hypotheses that $f$ and $g$ are convex and $f \equiv g$, or $f$ and $g$ sufficiently close with $u_{1}, u_{2}, u_{3}$ and $u_{4}$ satisfying a restrictive condition. He also calculated an example and obtained a very interesting numerical configuration of a solution which we shall construct and shall be called an envelope rarefaction wave. [5] deals also with equation (1) when $f \equiv g$, but with more general initial data piecewise constant in a finite number of wedges focused on the point $(0,0)$ in the $(x, y)$-plane. For the case $f \equiv g$, equation (1) can be transformed into $\partial u / \partial t+\partial(2 f) / \partial x^{\prime}=0$ through the coordinate transformation $x^{\prime}=x+y$, $y^{\prime}=x-y$, and problem (1), (2) is, in fact, a one-dimensional problem which can be handled easily. By contrast, it is easy to prove that equation (1) cannot be transformed into a one-dimensional equation through linear coordinate transformations whenever $\left(f^{\prime \prime} / g^{\prime \prime}\right)^{\prime} \neq 0$. We have obtained some more general results in the present paper. The entropy solutions to (1), (2) are constructed if $f$ and $g$ satisfy $f^{\prime \prime}(u) \neq 0, g^{\prime \prime}(u) \neq 0$ and $\left(f^{\prime \prime}(u) / g^{\prime \prime}(u)\right)^{\prime} \neq 0$, and these solutions $u$ are piecewise smooth with the upper and lower bounds unchanged, and consist of constant states, piecewise smooth discontinuity surfaces, centered planar wave bodies and centered wave cones.

The condition $\left(f^{\prime \prime}(u) / g^{\prime \prime}(u)\right)^{\prime} \neq 0$ is natural according to the point of view taken in [8]. In that paper the authors have worked out the concepts of convex and nonconvex and corresponding entropy condition in multidimensional space. Their results show that it is impossible for a single conservation law in two space dimensions to be either convex or concave in all directions. On the other hand $\left(f^{\prime \prime} / g^{\prime \prime}\right)^{\prime} \equiv 0$ means the equation is either convex or concave in all directions except one single direction (in this direction the equation degenerates into a linear one); in fact the equation will degenerate into a linear one if it is degenerate in more than one direction. It is, therefore, the simplest case to require that equation (1) has at most one inflection point in any specific direction. That is the condition $\left(f^{\prime \prime} / g^{\prime \prime}\right)^{\prime} \neq 0$. Incidentally, with a finer analysis, this condition can be removed without too much change in the method used; we assume this condition merely for simplicity.

In $\S 2$, we do some preliminaries. Through similarity transformations $\xi=$ $x / t, \eta=y / t$, problem (1), (2) then becomes a boundary value problem at infinity for an ordinary differential equation in the $(\xi, \eta)$-plane. In $\S 3$, we classify problem (1), (2) and solve the simplest case. In the remaining sections, we consider all other cases.

\section{Preliminaries}

For the Cauchy problem

$$
\begin{gathered}
\frac{\partial u}{\partial t}+\frac{\partial f(u)}{\partial x}+\frac{\partial g(u)}{\partial y}=0 \\
\left.u\right|_{t=0}=u_{0}(x, y),
\end{gathered}
$$


where $f$ and $g \in C^{3}(-\infty,+\infty)$ and $u_{0}(x, y)$ is a bounded measurable function, Kruzkov [3] has obtained the existence and uniqueness of weak solutions; these are defined as follows:

Definition. A bounded measurable function $u(t, x, y)$ is a weak solution of (1), (3) in the strip $\Pi_{T}=[0, T) \times R^{2}$ if

$$
\iiint_{\Pi_{T}}\left[u \frac{\partial \phi}{\partial t}+f(u) \frac{\partial \phi}{\partial x}+g(u) \frac{\partial \phi}{\partial y}\right] d x d y d t+\iint_{t=0} \phi \cdot u_{0}(x, y) d x d y=0
$$

for all $\phi \in C_{0}^{\infty}\left(\Pi_{T}\right)$. A function is a weak solution in the large if it is a weak solution for all $T>0$. The entropy condition satisfied by this weak solution is

$$
\begin{aligned}
\iiint_{\Pi_{T}} \operatorname{Sign}(u-k)\left\{(u-k) \frac{\partial \phi}{\partial t}+(f(u)-f(k))\right. & \frac{\partial \phi}{\partial x} \\
& \left.+(g(u)-g(k)) \frac{\partial \phi}{\partial y}\right\} d x d y \text { at } \geq 0
\end{aligned}
$$

for all real constants $k$ and any $\phi \in C_{0}^{\infty}\left(\Pi_{T}\right)$ such that $\phi \geq 0$.

For the Riemann problem (1), (2), we can obtain the existence and uniqueness of solutions using the results of Kruzkov. However, we shall explicitly construct these solutions in the class of piecewise smooth functions. In order to do this, we use the similarity transformation

$$
\xi=x / t, \quad \eta=y / t
$$

Let $u(t, x, y)=\tilde{u}(\xi, \eta)$. When $u \in C^{1}$, we have

$$
\begin{gathered}
u_{t}=\tilde{u}_{\xi} \cdot \frac{\partial \xi}{\partial t}+\tilde{u}_{\eta} \cdot \frac{\partial \eta}{\partial t}=-\frac{\xi}{t} \tilde{u}_{\xi}-\frac{\eta}{t} \tilde{u}_{\eta}, \\
u_{x}=\tilde{u}_{\xi} \frac{\partial \xi}{\partial x}=\frac{1}{t} \cdot \tilde{u}_{\xi}, \quad u_{y}=\frac{1}{t} \tilde{u}_{\eta}
\end{gathered}
$$

except $t=0$. Hence equation (1) becomes

$$
\left(f^{\prime}(\tilde{u})-\xi\right) \tilde{u}_{\xi}+\left(g^{\prime}(\tilde{u})-\eta\right) \tilde{u}_{\eta}=0
$$

and the initial data become

$$
\lim _{\substack{\xi / \eta=\text { const. } \\ \xi^{2}+\eta^{2} \rightarrow \infty}} \tilde{u}(\xi, \eta)= \begin{cases}u_{1}, & \text { for } \xi>0, \eta>0, \\ u_{2}, & \text { for } \xi<0, \eta>0, \\ u_{3}, & \text { for } \xi<0, \eta<0, \\ u_{4}, & \text { for } \xi>0, \eta<0 .\end{cases}
$$

For piecewise smooth solutions to problem (1), (2) with $S$ the discontinuity surface, $S$ must be a cone through the origin $(0,0,0)$, for reasons of selfsimilarity. Suppose $u^{-}$and $u^{+}\left(u^{-}<u^{+}\right)$are the values of $u$ on both sides of $S$, and the $\vec{n}$ is the normal to $S$ from $u^{-}$to $u^{+}$. Then the RankineHugoniot $(\mathrm{R}-\mathrm{H})$ relation and the entropy condition are

$$
\vec{n} \cdot\left(u^{+}-u^{-}, f\left(u^{+}\right)-f\left(u^{-}\right), g\left(u^{+}\right)-g\left(u^{-}\right)\right)=0
$$


and

$$
\vec{n} \cdot\left(k-u^{-}, f(k)-f\left(u^{-}\right), g(k)-g\left(u^{-}\right)\right) \geq 0
$$

respectively, for any constant $k$ such that $u^{-} \leq k \leq u^{+}$; these can be easily deduced from the definition of a solution.

Using the transformation (4), we obtain the admissible condition for discontinuity lines in the $(\xi, \eta)$-plane as follows. First,

$$
\frac{d \eta}{d \xi}=\left(\frac{g\left(u^{+}\right)-g\left(u^{-}\right)}{u^{+}-u^{-}}-\eta\right) /\left(\frac{f\left(u^{+}\right)-f\left(u^{-}\right)}{u^{+}-u^{-}}-\xi\right) .
$$

Second, assuming that $(\alpha, \beta)$ is the projection of $\vec{n}$ onto the $(\xi, \eta)$-plane, then

$$
\frac{F(k)-F\left(u^{-}\right)}{k-u^{-}} \geq \frac{F\left(u^{+}\right)-F\left(u^{-}\right)}{u^{+}-u^{-}}
$$

for any constant $k$ such that $u^{-} \leq k \leq u^{+}$, where $F=\alpha f+\beta g$.

Obviously, any piecewise smooth function $\tilde{u}(\xi, \eta)$ in $R^{2}$ having piecewise discontinuity lines on which (9), (10) are satisfied, satisfying (5) in smooth regions and satisfying (6) in the infinity, is bound to be the unique weak solution to the Riemann problem (1), (2) in the sense of Kruzkov after inverting the transformation (4).

When we rewrite equation (5) in another form,

$$
(f(\tilde{u})-\xi \tilde{u})_{\xi}+(g(\tilde{u})-\eta \tilde{u})_{\eta}=-2 \tilde{u},
$$

we find that (9), (10) are just the R-H relation and the entropy condition for discontinuity lines for equation $\left(5^{\prime}\right)$. Thus, we still call the discontinuities satisfying (9), (10) shocks or contact discontinuities. We shall omit " " on $u$ for brevity.

What we want to do is construct the piecewise smooth solutions to equation (5) with boundary values (6), satisfying admissible discontinuity conditions (9), (10). Let us consider first the smooth solutions to equation (5).

Equation (5) can be written in characteristic form

$$
\left\{\begin{array}{l}
\frac{d u(\xi, \eta(\xi))}{d \xi}=0, \\
\frac{d \eta(\xi)}{d \xi}=\frac{g^{\prime}(u)-\eta}{f^{\prime}(u)-\xi}
\end{array}\right.
$$

The characteristic lines are obviously any half rays starting at $\left(f^{\prime}(u), g^{\prime}(u)\right)$ along which $u$ is constant. Therefore, we can easily obtain the continuous solutions of the above equations.

Thus the curve

$$
\left\{\begin{array}{l}
\xi=f^{\prime}(u), \\
\eta=g^{\prime}(u), \quad(-\infty<u<+\infty)
\end{array}\right.
$$

with $u$ as parameter plays an important role. We call it a base curve and denote it by (B). It is easy to show that the base curve is monotonically increasing and concave under the condition $f^{\prime \prime}>0, g^{\prime \prime}>0$ and $\left(f^{\prime \prime} / g^{\prime \prime}\right)^{\prime}>0$. 


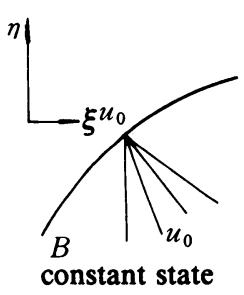

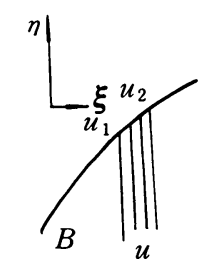

one-dimensional planar wave

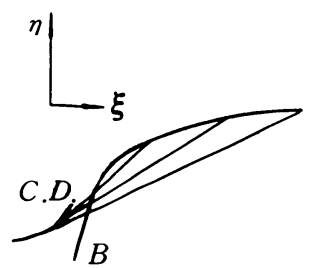

envelope rarefaction wave

FIGURE 2.1

If we now consider piecewise smooth solutions, we can also construct the general configurations of the solutions which consist of domains of constant states, regions of nonconstant continuous solutions with characteristic lines or admissible discontinuity lines as boundaries. For problem (5), (6), (9), (10) under the hypothesis $f^{\prime \prime} g^{\prime \prime}\left(f^{\prime \prime} / g^{\prime \prime}\right)^{\prime} \neq 0$ we shall show that continuous solutions take at most three forms (see Figure 2.1); one form is the constant state, the second is a one-dimensional planar wave, and the third is foliated by characteristic lines generated tangentially from a half contact discontinuity. We call the last form of solution an envelope rarefaction wave. When we transform these solutions back into the $(t, x, y)$-plane, we shall call them constant states, centered planar wave bodies, and contered wave cones, respectively (see Figure 2.1).

\section{Classification and Simple Cases}

We simplify the general Riemann problem (1), (2) under the condition

$$
f^{\prime \prime} g^{\prime \prime}\left(f^{\prime \prime} / g^{\prime \prime}\right)^{\prime} \neq 0
$$

as follows. First, we may assume $f^{\prime \prime}>0$ and $g^{\prime \prime}>0$ without loss of generality; otherwise we may use one of the transformations $x \rightarrow-x, y \rightarrow-y ; x \rightarrow-x$, $y \rightarrow y$; or $x \rightarrow x, y \rightarrow-y$. Next we can assume $\left(f^{\prime \prime} / g^{\prime \prime}\right)^{\prime}>0$; otherwise we may uses the transformation $x \rightarrow-x, y \rightarrow-y, u \rightarrow-u$ without changing the signs of $f^{\prime \prime}$ and $g^{\prime \prime}$. Also we can assume $u_{2} \leq u_{4}$; otherwise we may use the transformation $x \rightarrow-y, y \rightarrow-x, u \rightarrow-u$ leaving the signs of $f^{\prime \prime}$, $g^{\prime \prime}$ and $\left(f^{\prime \prime} / g^{\prime \prime}\right)^{\prime}$ unchanged. Thus we need only solve the Riemann problem under the hypotheses $f^{\prime \prime}>0, g^{\prime \prime}>0,\left(f^{\prime \prime} / g^{\prime \prime}\right)^{\prime}>0$ and $u_{2} \leq u_{4}$.
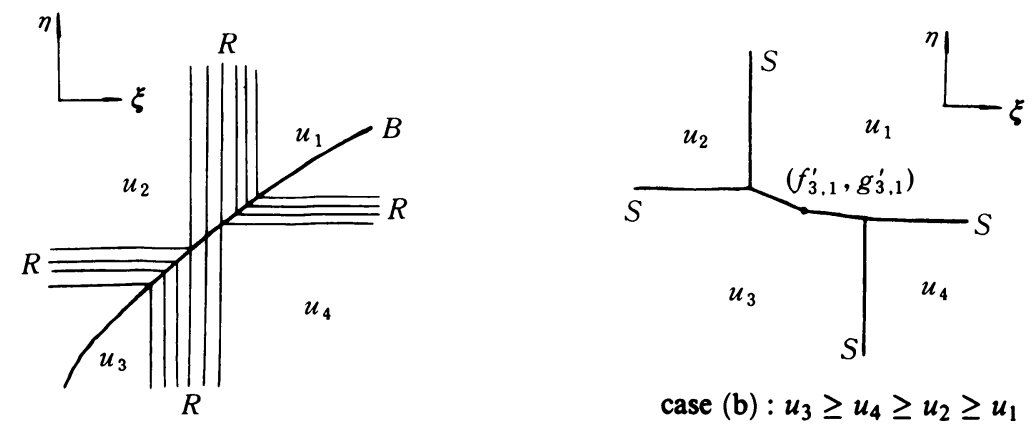

$$
\text { case (a): } u_{3} \leq u_{2} \leq u_{4} \leq u_{1}
$$

case (b) : $u_{3} \geq u_{4} \geq u_{2} \geq u_{1}$

FIGURE 3.1 
Solutions to problem (5), (6) must be four one-dimensional planar waves outside a bounded domain in $(\xi, \eta)$-plane. We can use these "exterior" waves to classify problem (5), (6) into five cases: (a) no shocks, (b) no rarefaction waves, (c) exactly one shock, (d) exactly one rarefaction wave, and (e) two rarefaction waves and two shocks. We can easily construct the solutions to (a) and (b) (Figure 3.1). There must be $u_{3} \leq u_{2} \leq u_{4} \leq u_{1}$ or $u_{3} \geq u_{4} \geq u_{2} \geq u_{1}$ in cases (a) and (b) respectively. The interior shocks in case (b) satisfy the R-H relation for $u^{-}=u_{1}, u^{+}=u_{3}$ :

$$
\frac{d \eta}{d \xi}=\frac{g_{3,1}^{\prime}-\eta}{f_{3,1}^{\prime}-\xi}, \quad \text { where } f_{i, j}^{\prime}=\frac{f\left(u_{i}\right)-f\left(u_{j}\right)}{u_{i}-u_{j}} .
$$

The solutions to this equation are half rays starting at the point $\left(f_{3,1}^{\prime}, g_{3,1}^{\prime}\right)$. The entropy condition is obviously satisfied because the normals to the two straight segments in the middle of Figure 3.1, case (b) make $F$ concave. We shall consider the remaining cases in the following sections.

\section{CASE (c): EXACTLY ONE SHOCK}

In this case there are only two possible orderings of initial data: $\left(\mathrm{c}_{1}\right): u_{4}>$ $u_{1} \geq u_{2} \geq u_{3}$ or $\left(\mathrm{c}_{2}\right): u_{2}<u_{3} \leq u_{4} \leq u_{1}$. We can construct the solutions as in Figure 4.1.

Case $\left(c_{1}\right)$. It is sufficient to consider the case $u_{4}>u_{1}=u_{2}=u_{3}$. In Figure 4.2, the wave parallel to the $\eta$-axis is a rarefaction wave $\xi=f^{\prime}(u), u_{1} \leq u \leq u_{4}$, and the wave parallel to the $\xi$-axis is a shock $\eta=g_{1,4}^{\prime}$. We need to construct the solution in the rectangle (Figure 4.2).

We solve the R-H relation first from the point $A\left(f^{\prime}\left(u_{4}\right), g_{1,4}^{\prime}\right)$ :

$$
\left\{\begin{array}{l}
\frac{d \eta}{d \xi}=\left(\frac{g\left(u_{1}\right)-g(u)}{u_{1}-u}-\eta\right) /\left(\frac{f\left(u_{1}\right)-f(u)}{u_{1}-u}-\xi\right), \quad \xi=f^{\prime}(u), \\
\left.\eta\right|_{\xi=f^{\prime}\left(u_{4}\right)}=g_{1,4}^{\prime}
\end{array}\right.
$$

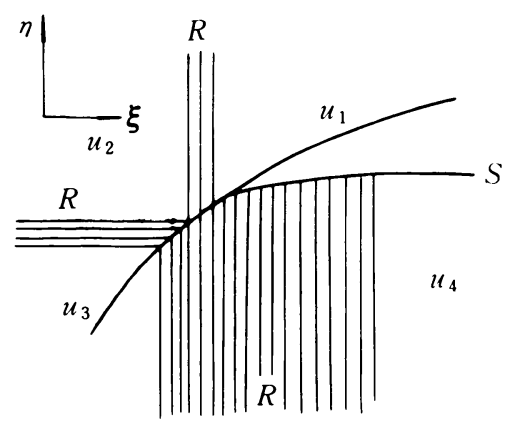

case $\left(c_{1}\right): u_{4}>u_{1} \geq u_{2} \geq u_{3}$

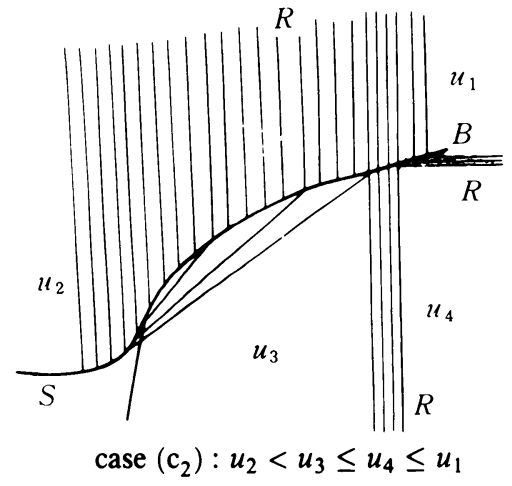

FIGURE 4.1 


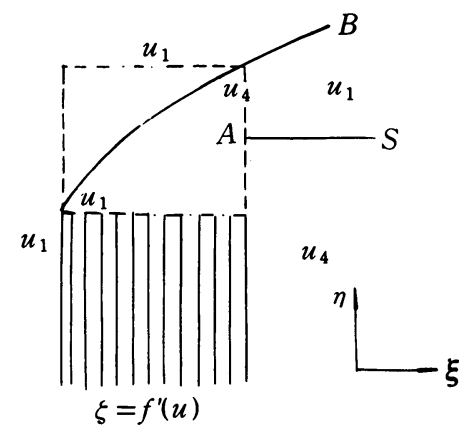

Figure 4.2

with $u^{+}=u, u^{-}=u_{1}$ as values of $u$ on both sides of the discontinuity. The solution can easily be found. Let

$$
a\left(\lambda, u_{1}\right)=f^{\prime \prime}(\lambda) /\left(\frac{f\left(u_{1}\right)-f(\lambda)}{u_{1}-\lambda}-f^{\prime}(\lambda)\right) ;
$$

then the solution takes the form

$$
\left\{\begin{aligned}
\xi=f^{\prime}(u), & \\
\eta=\eta_{s}(u) \equiv & \int_{u_{4}}^{u} a\left(s, u_{1}\right) \frac{g(s)-g\left(u_{1}\right)}{s-u_{1}} \exp \left(\int_{u}^{s} a\left(\lambda, u_{1}\right) d \lambda\right) d s \\
& +g_{1,4}^{\prime} \exp \left(-\int_{u_{1}}^{u} a\left(\lambda, u_{1}\right) d \lambda\right), \quad u_{1}<u \leq u_{4} .
\end{aligned}\right.
$$

It is easy to prove that this integral curve is monotonically increasing in $\left(u_{1}, u_{4}\right)$. In fact, we have

$$
\begin{aligned}
& \frac{g(u)-g\left(u_{1}\right)}{u-u_{1}}-\eta_{s}(u)= \exp \left(-\int_{u_{4}}^{u} a\left(\lambda, u_{1}\right) d \lambda\right) \\
& \cdot\left[\frac{g(u)-g\left(u_{1}\right)}{u-u_{1}} \cdot \exp \left(\int_{u_{4}}^{u} a\left(\lambda, u_{1}\right) d \lambda\right)-g_{1,4}^{\prime}\right. \\
&\left.\quad-\int_{u_{4}}^{u} a\left(s, u_{1}\right) \frac{g(s)-g\left(u_{1}\right)}{s-u_{1}} \exp \left(\int_{u_{4}}^{s} a\left(\lambda, u_{1}\right) d \lambda\right) d s\right] \\
& \stackrel{\substack{\text { initegrate } \\
\text { by parts) }}}{=} \int_{u_{4}}^{u} \exp \left(\int_{u}^{s} a\left(\lambda, u_{1}\right) d \lambda\right) \frac{d}{d s}\left(\frac{g(s)-g\left(u_{1}\right)}{s-u_{1}}\right) d s<0,
\end{aligned}
$$

and

$$
\frac{f(u)-f\left(u_{1}\right)}{u-u_{1}}-f^{\prime}(u)<0 \quad\left(u_{1}<u \leq u_{4}\right) .
$$

Therefore, we have

$$
d \eta_{s} / d \xi>0, \quad \xi \in\left(f^{\prime}\left(u_{1}\right), f^{\prime}\left(u_{4}\right)\right)
$$


In order to prove that the integral curve intersects the base curve at $\left(f^{\prime}\left(u_{1}\right)\right.$, $g^{\prime}\left(u_{1}\right)$ ) tangentially (and also for later use), we prove that

$$
\begin{array}{r}
\eta_{s}(u)=g^{\prime}(u)+O\left(\left(u-u_{1}\right)^{2}\right)-\frac{1}{3}\left(\frac{g^{\prime \prime}(u)}{f^{\prime \prime}(u)}\right)_{u=u_{1}}^{\prime} \cdot f^{\prime \prime}\left(u_{1}\right)\left(u-u_{1}\right)^{2} \ln \left(u-u_{1}\right), \\
\left(u \rightarrow u_{1}+0\right) .
\end{array}
$$

Here, we assume $f^{\prime \prime \prime}$ and $g^{\prime \prime \prime}$ are bounded when $u$ is bounded.

Using a Taylor expansion, we get

$$
\begin{aligned}
f\left(u_{1}\right)= & f(\lambda)+f^{\prime}(\lambda)\left(u_{1}-\lambda\right)+\frac{f^{\prime \prime}(\lambda)}{2}\left(u_{1}-\lambda\right)^{2} \\
& +\frac{f^{\prime \prime \prime}(\lambda)}{6}\left(u_{1}-\lambda\right)^{3}+O\left(\left(\lambda-u_{1}\right)^{4}\right)
\end{aligned}
$$

therefore

$$
\begin{aligned}
a\left(\lambda, u_{1}\right) & =f^{\prime \prime}(\lambda) /\left(\frac{f\left(u_{1}\right)-f(\lambda)}{u_{1}-\lambda}-f^{\prime}(\lambda)\right) \\
& =1 /\left(\frac{u_{1}-\lambda}{2}+\frac{f^{\prime \prime \prime}(\lambda)}{6 f^{\prime \prime}(\lambda)}\left(u_{1}-\lambda\right)^{2}+O\left(\left(\lambda-u_{1}\right)^{3}\right)\right) \\
& =\frac{2}{u_{1}-\lambda}-\frac{2}{3} \frac{f^{\prime \prime \prime}(\lambda)}{f^{\prime \prime}(\lambda)}+O\left(\left(\lambda-u_{1}\right)\right), \quad \lambda>u_{1} .
\end{aligned}
$$

Then

$$
\begin{aligned}
& \exp \left(\int_{u}^{s} a\left(\lambda, u_{1}\right) d \lambda\right) \\
& =\exp \left(-2 \ln \frac{s-u_{1}}{u-u_{1}}-\frac{2}{3} \ln \frac{f^{\prime \prime}(s)}{f^{\prime \prime}(u)}+O\left(\left(s-u_{1}\right)^{2}-\left(u-u_{1}\right)^{2}\right)\right) \\
& =\left(\frac{u-u_{1}}{s-u_{1}}\right)^{2}\left(\frac{f^{\prime \prime}(u)}{f^{\prime \prime}(s)}\right)^{2 / 3} \exp \left(O\left(s-u_{1}\right)^{2}+\left(u-u_{1}\right)^{2}\right) \\
& =\left(\frac{u-u_{1}}{s-u_{1}}\right)^{2}\left(\left(f^{\prime \prime}\left(u_{1}\right)\right)^{2 / 3}+O\left(u-u_{1}\right)\right) \\
& \cdot\left(\frac{1}{\left(f^{\prime \prime}\left(u_{1}\right)\right)^{2 / 3}}-\frac{2}{3} \frac{f^{\prime \prime \prime}\left(u_{1}\right)}{\left(f^{\prime \prime}\left(u_{1}\right)\right)^{5 / 3}}\left(s-u_{1}\right)+O\left(\left(s-u_{1}\right)^{2}\right)\right) \\
& \cdot\left(1+O\left(\left(u-u_{1}\right)^{2}\right)\right) \cdot\left(1+O\left(\left(s-u_{1}\right)^{2}\right)\right) \\
& =\left(\frac{u-u_{1}}{s-u_{1}}\right)^{2}\left(1-\frac{2}{3} \frac{f^{\prime \prime \prime}\left(u_{1}\right)}{f^{\prime \prime}\left(u_{1}\right)}\left(s-u_{1}\right)+O\left(\left(s-u_{1}\right)^{2}\right)\right) \\
& \cdot\left(1+O\left(u-u_{1}\right)\right) \text {. }
\end{aligned}
$$


Thus,

$$
\begin{aligned}
& \int_{u_{4}}^{u} \exp \left(\int_{u}^{s} a\left(\lambda, u_{1}\right) d \lambda\right) d s \\
& \quad=\left(u-u_{1}\right)^{2}\left(1+O\left(u-u_{1}\right)\right)\left(\int_{u_{4}}^{u} \frac{d s}{\left(s-u_{1}\right)^{2}}-\frac{2}{3} \frac{f^{\prime \prime \prime}\left(u_{1}\right)}{f^{\prime \prime}\left(u_{1}\right)} \int_{u_{4}}^{u} \frac{d s}{s-u_{1}}+O(1)\right) \\
& \quad=\left(u-u_{1}\right)^{2}\left(1+O\left(u-u_{1}\right)\right)\left(\frac{1}{u_{1}-u}-\frac{2}{3} \frac{f^{\prime \prime \prime}\left(u_{1}\right)}{f^{\prime \prime}\left(u_{1}\right)} \ln \left(u-u_{1}\right)+O(1)\right) \\
& =\left(u_{1}-u\right)-\frac{2}{3} \frac{f^{\prime \prime \prime}\left(u_{1}\right)}{f^{\prime \prime}\left(u_{1}\right)}\left(u-u_{1}\right)^{2} \ln \left(u-u_{1}\right)+O\left(\left(u-u_{1}\right)^{2}\right)
\end{aligned}
$$

and

$$
\begin{aligned}
\int_{u_{4}}^{u}(s & \left.-u_{1}\right) \exp \left(\int_{u}^{s} a\left(\lambda, u_{3}\right) d \lambda\right) d s \\
= & \left(u-u_{1}\right)^{2}\left(1+O\left(u-u_{1}\right)\right)\left(\int_{u_{4}}^{u} \frac{d s}{s-u_{1}}+O(1)\right) \\
= & \left(u-u_{1}\right)^{2} \ln \left(u-u_{1}\right)+O\left(\left(u-u_{1}\right)^{2}\right) .
\end{aligned}
$$

Therefore,

$$
\begin{aligned}
\int_{u_{4}}^{u} \exp \left(\int_{u}^{s} a\left(\lambda, u_{1}\right) d \lambda\right) \frac{d}{d s}\left(\frac{g(s)-g\left(u_{1}\right)}{s-u_{1}}\right) d s \\
=\int_{u_{4}}^{u} \exp \left(\int_{u}^{s} a\left(\lambda, u_{1}\right) d \lambda\right)\left(\frac{g^{\prime \prime}\left(u_{1}\right)}{2}+\frac{g^{\prime \prime \prime}\left(u_{1}\right)}{3}\left(s-u_{1}\right)+O\left(\left(s-u_{1}\right)^{2}\right)\right) d s \\
=\frac{g^{\prime \prime}\left(u_{1}\right)}{2}\left(u_{1}-u\right)+\left(-\frac{g^{\prime \prime}\left(u_{1}\right) f^{\prime \prime \prime}\left(u_{1}\right)}{3 f^{\prime \prime}\left(u_{1}\right)}+\frac{g^{\prime \prime \prime}\left(u_{1}\right)}{3}\right) \\
\quad \times\left(u-u_{1}\right)^{2} \ln \left(u-u_{1}\right)+O\left(\left(u-u_{1}\right)^{2}\right) \\
=-\frac{1}{2} g^{\prime \prime}\left(u_{1}\right)\left(u-u_{1}\right)+\left.\frac{1}{3}\left(\frac{g^{\prime \prime}}{f^{\prime \prime}}\right)^{\prime} f^{\prime \prime}\right|_{u_{1}}\left(u-u_{1}\right)^{2} \ln \left(u-u_{1}\right)+O\left(\left(u-u_{1}\right)^{2}\right) .
\end{aligned}
$$

Finally we get

$$
\begin{aligned}
\eta_{s}= & \frac{g(u)-g\left(u_{1}\right)}{u-u_{1}}-\int_{u_{4}}^{u} \exp \left(\int_{u}^{s} a\left(\lambda, u_{1}\right) d \lambda\right) \frac{d}{d s}\left(\frac{g(s)-g\left(u_{1}\right)}{s-u_{1}}\right) d s \\
= & g^{\prime}\left(u_{1}\right)+\frac{g^{\prime \prime}\left(u_{1}\right)}{2}\left(u-u_{1}\right)+\frac{g^{\prime \prime}\left(u_{1}\right)}{2}\left(u-u_{1}\right) \\
& -\left.\frac{1}{3}\left(\frac{g^{\prime \prime}(u)}{f^{\prime \prime}(u)}\right)^{\prime}\right|_{u_{1}} \cdot f^{\prime \prime}\left(u_{1}\right)\left(u-u_{1}\right)^{2} \ln \left(u-u_{1}\right)+O\left(\left(u-u_{1}\right)^{2}\right) \\
= & g^{\prime}(u)-\left.\frac{1}{3}\left(\frac{g^{\prime \prime}}{f^{\prime \prime}}\right)^{\prime}\right|_{u_{1}} f^{\prime \prime}\left(u_{1}\right)\left(u-u_{1}\right)^{2} \ln \left(u-u_{1}\right)+O\left(\left(u-u_{1}\right)^{2}\right) .
\end{aligned}
$$


This results in

$$
\begin{gathered}
\lim _{u \rightarrow u_{1}} \eta_{s}(u)=g^{\prime}\left(u_{1}\right) \\
\lim _{u \rightarrow u_{1}} \frac{d \eta_{s}}{d \xi}=\frac{g^{\prime \prime}\left(u_{1}\right)}{f^{\prime \prime}\left(u_{1}\right)}=\text { the slope of the base curve at } u_{1} .
\end{gathered}
$$

To prove $\eta=\eta_{s}(u)<g^{\prime}(u)$ in the interval $\left(u_{1}, u_{4}\right]$, i.e., that the integral curve is always under the base curve, we prove first the following inequality:

(A)

$$
\left(\frac{g(u)-g\left(u_{1}\right)}{u-u_{1}}-g^{\prime}(u)\right) /\left(\frac{f(u)-f\left(u_{1}\right)}{u-u_{1}}-f^{\prime}(u)\right)>\frac{g^{\prime \prime}(u)}{f^{\prime \prime}(u)} \quad\left(u>u_{1}\right) .
$$

In fact, if we define

$$
F\left(u, u_{1}\right) \equiv\left(g(u)-g\left(u_{1}\right)-g^{\prime}(u)\left(u-u_{1}\right)\right)-\left(f(u)-f\left(u_{1}\right)-f^{\prime}(u)\left(u-u_{1}\right)\right) \frac{g^{\prime \prime}(u)}{f^{\prime \prime}(u)} \text {, }
$$

then

$$
F\left(u_{1}, u_{1}\right)=0, \quad \partial F / \partial u=-\left(f(u)-f\left(u_{1}\right)-f^{\prime}(u)\left(u-u_{1}\right)\right)\left(g^{\prime \prime}(u) / f^{\prime \prime}(u)\right)^{\prime}<0,
$$

so inequality $(\mathrm{A})$ holds.

We want to show that the integral curve is always under the base curve. In fact, we first have $\eta=g_{1,4}^{\prime}<g^{\prime}\left(u_{4}\right)$. We then suppose there is a $\bar{u} \in\left(u_{1}, u_{4}\right)$ such that $\eta=\eta_{s}(u)<g^{\prime}(u)$ holds in the interval $\left(\bar{u}, u_{4}\right]$, and that $\eta=\eta_{s}(\bar{u})=$ $g^{\prime}(\bar{u}) ;$ then

$$
\left.\frac{d \eta_{s}}{d \xi}\right|_{\bar{u}} \leq \frac{g^{\prime \prime}(\bar{u})}{f^{\prime \prime}(\bar{u})}
$$

because $d \eta_{s} / d \xi \neq \infty$ in the interval $\left(u_{1}, u_{4}\right)$; namely,

$$
\left(\frac{g(\bar{u})-g\left(u_{1}\right)}{\bar{u}-u_{1}}-g^{\prime}(\bar{u})\right) /\left(\frac{f(\bar{u})-f\left(u_{1}\right)}{\bar{u}-u_{1}}-f^{\prime}(\bar{u})\right) \leq \frac{g^{\prime \prime}(\bar{u})}{f^{\prime \prime}(\bar{u})}, \quad u_{1}<\bar{u}<u_{4},
$$

which is impossible according to inequality (A). Therefore the integral curve is always under the base curve.

Thus,

$$
\frac{d}{d \xi}\left(\frac{d \eta_{s}}{d \xi}\right)=\frac{1}{f^{\prime \prime}(u)} \frac{1 /\left(u-u_{1}\right)}{f^{\prime}(u)-\left(f(u)-f\left(u_{1}\right)\right) /\left(u-u_{1}\right)}\left(\eta_{s}(u)-g^{\prime}(u)\right)<0,
$$

so $\eta=\eta_{s}(\xi)$ is a concave curve.

There exists a neighbourhood of the point $\left(f^{\prime}\left(u_{4}\right), g_{1,4}^{\prime}\right)$ in which $\eta=\eta_{s}(\xi)$ satisfies the entropy condition (10). In fact, the normal to $\eta=\eta_{s}(\xi)$ may be taken as $\left(1,-d \xi / d \eta_{s}\right)$ pointing from $u_{1}$ to $u$, and $F(v)=f(v)-$ $\left(d \xi / d \eta_{s}\right) g(v)$. Noting that $F_{\alpha}(v)=f(v)+\alpha g(v)$ has at most one inflection point for any $\alpha,-\infty<\alpha<+\infty$, we may denote this inflection point by $\stackrel{\circ}{u}(\alpha)$. Then $\stackrel{\circ}{u}(\alpha)$ is a monotonically decreasing function in an interval $\subset(-\infty, 0)$ and unbounded from both sides (Figure 4.3). Therefore there exists an $M>0$ 
such that when $\alpha<-M, \stackrel{\circ}{u}(\alpha)>u_{4}$ holds. As $d \eta_{s} /\left.d \xi\right|_{\xi=f^{\prime}\left(u_{4}\right)}=0$, we can choose an $\varepsilon>0$ such that

$$
0 \leq \frac{d \eta_{s}}{d \xi}<\frac{1}{M}, \quad \xi \in\left(f^{\prime}\left(u_{4}\right)-\varepsilon, f^{\prime}\left(u_{4}\right)\right]
$$

Thus, in this neighbourhood, the discontinuity line $\eta=\eta_{s}(\xi)$ with $u^{-}=u_{1}$, $u^{+}=u \leq u_{4}$, satisfies the entropy condition (10).

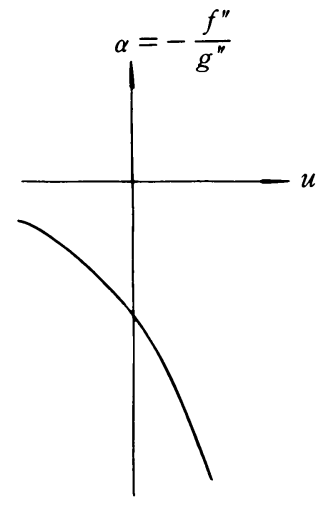

FIGURE 4.3

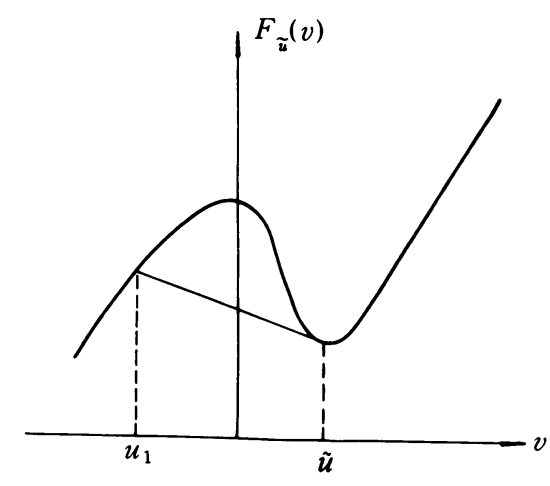

FIGURE 4.4

We next prove $\eta=\eta_{s}(\xi)$ satisfies (10) until $u=u_{1}$. Otherwise there exists a $\tilde{u}>u_{1}$, where

$$
\tilde{u}=\inf \left\{\bar{u} \mid \eta_{s}(\xi) \text { satisfies }(10) \text { in }\left[\bar{u}, u_{4}\right]\right\} \text {. }
$$

Then, at $u=\tilde{u}, \eta_{s}(\xi)$ satisfies (10), too. Noting that $d^{2} \eta_{s} / d \xi^{2}<0$ and $\stackrel{\circ}{u}(-d \xi / d \eta)$ decreases from $+\infty$ with $\xi$ decreasing from $f^{\prime}\left(u_{4}\right)$, we know that the entropy condition satisfied by $\eta=\eta_{s}(\xi)$ at $u=\tilde{u}$ must be of the form (Figure 4.4)

$$
\begin{aligned}
& \left.\frac{d F_{\tilde{u}}(v)}{d v}\right|_{v=\tilde{u}}=\frac{F_{\tilde{u}}(\tilde{u})-F_{\tilde{u}}\left(u_{1}\right)}{\tilde{u}-u_{1}}, \\
& F_{\tilde{u}}(v)=f(v)-\left.\frac{d \xi}{d \eta_{s}}\right|_{u=\tilde{u}} \cdot g(v) ;
\end{aligned}
$$

i.e.,

$$
\left.\frac{d \eta_{s}}{d \xi}\right|_{u=\tilde{u}}=\left(g^{\prime}(\tilde{u})-\frac{g(\tilde{u})-g\left(u_{1}\right)}{\tilde{u}-u_{1}}\right) /\left(f^{\prime}(\tilde{u})-\frac{f(\tilde{u})-f\left(u_{1}\right)}{\tilde{u}-u_{1}}\right) .
$$

This and the R-H relation result in $\eta_{s}(\tilde{u})=g^{\prime}(\tilde{u})$; i.e., $\tilde{u}=u_{1}$. This contradicts $\tilde{u}>u_{1}$. Thus the entire curve satisfies the entropy condition (10).

We may now obtain Figure 4.1, case $\left(c_{1}\right)$ in which the shock is a concave curve ending tangentially with the base curve at the point $\left(f^{\prime}\left(u_{1}\right), g^{\prime}\left(u_{1}\right)\right)$. 
Case $\left(c_{2}\right)$. As before, we solve first the $\mathrm{R}-\mathrm{H}$ relation

$$
\left\{\begin{array}{l}
\frac{d \eta_{s}}{d \xi}=\left(\frac{g(u)-g\left(u_{3}\right)}{u-u_{3}}-\eta\right) /\left(\frac{f(u)-f\left(u_{3}\right)}{u-u_{3}}-\xi\right), \quad \xi=f^{\prime}(u), \\
\left.\eta\right|_{\xi=f^{\prime}\left(u_{2}\right)}=g_{2,3}^{\prime}
\end{array}\right.
$$

to get

$$
\begin{aligned}
\eta= & \eta_{s}(u) \equiv g_{2,3}^{\prime} \exp \left(-\int_{u_{2}}^{u} a\left(\lambda, u_{3}\right) d \lambda\right) \\
& \quad+\int_{u_{2}}^{u} \exp \left(\int_{u}^{s} a\left(\lambda, u_{3}\right) d \lambda\right) \frac{g(s)-g\left(u_{3}\right)}{s-u_{3}} a\left(s, u_{3}\right) d s \\
\xi= & f^{\prime}(u), \quad u_{2} \leq u<u_{3},
\end{aligned}
$$

and

$$
\begin{gathered}
\lim _{u \rightarrow u_{3}} \eta_{s}(u)=g^{\prime}\left(u_{3}\right), \quad \frac{d \eta_{s}}{d \xi}>0 \quad\left(u_{2}<u<u_{3}\right), \\
\lim _{u \rightarrow u_{3}} \frac{d \eta_{s}}{d \xi}=\frac{g^{\prime \prime}\left(u_{3}\right)}{f^{\prime \prime}\left(u_{3}\right)}, \\
\frac{d^{2} \eta_{s}}{d \xi^{2}}=\frac{1}{f^{\prime \prime}(u)} \cdot \frac{1}{u_{3}-u} \cdot \frac{1}{\left(f\left(u_{3}\right)-f(u)\right) /\left(u_{3}-u\right)-f^{\prime}(u)} \cdot\left(\eta_{s}(u)-g^{\prime}(u)\right) \\
=-\left.\frac{2}{3} \frac{1}{f^{\prime \prime}\left(u_{3}\right)}\left(\frac{g^{\prime \prime}(u)}{f^{\prime \prime}(u)}\right)^{\prime}\right|_{u=u_{3}} \ln \left(u_{3}-u\right)+O(1) ;
\end{gathered}
$$

thus

$$
\lim _{u \rightarrow u_{3}} \frac{d^{2} \eta_{s}}{d \xi^{2}}=-\infty
$$

It is easy to conclude from this that the integral curve must intersect the base curve at a point $\bar{u} \in\left(u_{2}, u_{3}\right)$ (Figure 4.5).

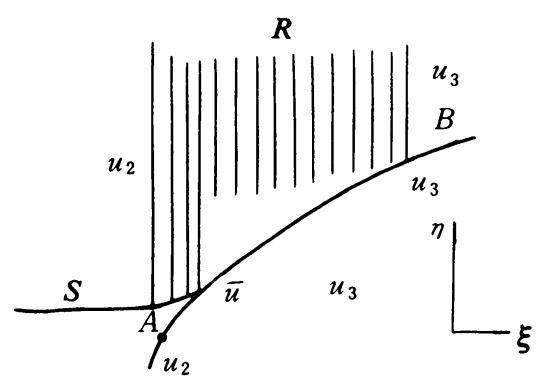

FIGURE 4.5 
At this stage, we first prove another inequality; namely,

(B) $\frac{g^{\prime}(u)-\left(g(u)-g\left(u_{1}\right)\right) /\left(u-u_{1}\right)}{f^{\prime}(u)-\left(f(u)-f\left(u_{1}\right)\right) /\left(u-u_{1}\right)}<\frac{g^{\prime}\left(u_{1}\right)-\left(g(u)-g\left(u_{1}\right)\right) /\left(u-u_{1}\right)}{f^{\prime}\left(u_{1}\right)-\left(f(u)-f\left(u_{1}\right)\right) /\left(u-u_{1}\right)}$

Proof. Let

$$
\left(u>u_{1}\right)
$$

$$
\begin{aligned}
F_{1}(u)= & \left(g^{\prime}(u)-\frac{g(u)-g\left(u_{1}\right)}{u-u_{1}}\right)\left(f^{\prime}\left(u_{1}\right)\left(u-u_{1}\right)-\left(f(u)-f\left(u_{1}\right)\right)\right) \\
& -\left(g^{\prime}\left(u_{1}\right)-\frac{g(u)-g\left(u_{1}\right)}{u-u_{1}}\right)\left(f^{\prime}(u)\left(u-u_{1}\right)-\left(f(u)-f\left(u_{1}\right)\right)\right) ;
\end{aligned}
$$

then $F_{1}\left(u_{1}\right)=0$, and

$$
\begin{aligned}
\frac{d F_{1}(u)}{d u}=f^{\prime \prime}(u)\left[\frac{g^{\prime \prime}(u)}{f^{\prime \prime}(u)}\left(f^{\prime}\left(u_{1}\right)\left(u-u_{1}\right)-\left(f(u)-f\left(u_{1}\right)\right)\right)\right. & \\
& \left.+g(u)-g\left(u_{1}\right)-g^{\prime}\left(u_{1}\right)\left(u-u_{1}\right)\right] .
\end{aligned}
$$

Let

$F_{2}(u)=\frac{g^{\prime \prime}(u)}{f^{\prime \prime}(u)}\left(f^{\prime}\left(u_{1}\right)\left(u-u_{1}\right)-\left(f(u)-f\left(u_{1}\right)\right)\right)+g(u)-g\left(u_{1}\right)-g^{\prime}\left(u_{1}\right)\left(u-u_{1}\right)$;

then $F_{2}\left(u_{1}\right)=0$, and

$$
\begin{aligned}
\frac{d F_{2}(u)}{d u}= & \left(\frac{g^{\prime \prime}(u)}{f^{\prime \prime}(u)}\right)^{\prime}\left(f^{\prime}\left(u_{1}\right)\left(u-u_{1}\right)-\left(f(u)-f\left(u_{1}\right)\right)\right) \\
& +\frac{g^{\prime \prime}(u)}{f^{\prime \prime}(u)}\left(f^{\prime}\left(u_{1}\right)-f^{\prime}(u)\right)+g^{\prime}(u)-g^{\prime}\left(u_{1}\right) .
\end{aligned}
$$

There exists a $\theta \in\left(u_{1}, u\right)$ such that

$$
\frac{g^{\prime}\left(u_{1}\right)-g^{\prime}(u)}{f^{\prime}\left(u_{1}\right)-f^{\prime}(u)}=\frac{g^{\prime \prime}(\theta)}{f^{\prime \prime}(\theta)}>\frac{g^{\prime \prime}(u)}{f^{\prime \prime}(u)}
$$

Therefore

$$
\frac{g^{\prime \prime}(u)}{f^{\prime \prime}(u)}\left(f^{\prime}\left(u_{1}\right)-f^{\prime}(u)\right)+g^{\prime}(u)-g^{\prime}\left(u_{1}\right)>0 .
$$

This completes the proof of inequality (B).

Suppose $\bar{u}=\sup \left\{u \mid u_{2} \leq u \leq u_{3}\right.$ and $\eta_{s}(v)>g^{\prime}(v)$ for $\left.v \in\left(u_{2}, u\right)\right\}$; then $\bar{u}<u_{3}$. We consider the point $u^{*}=\sup \left\{u^{* *} \mid \eta_{s}(u)\right.$ satisfies $(10)$ in $\left(u_{2}, u^{* *}\right)$, $\left.u^{* *} \leq \bar{u}\right\}$. There are only two possibilities; i.e.,

$$
F_{\alpha}^{\prime}\left(u^{*}\right)=\frac{F_{\alpha}\left(u^{*}\right)-F_{\alpha}\left(u_{3}\right)}{u^{*}-u_{3}}, \quad \alpha=-\frac{d \xi}{d \eta}\left(u^{*}\right),
$$

or

$$
F_{\alpha}^{\prime}\left(u_{3}\right)=\frac{F_{\alpha}\left(u^{*}\right)-F_{\alpha}\left(u_{3}\right)}{u^{*}-u_{3}}
$$


The former is equivalent to $\eta_{s}\left(u^{*}\right)=g^{\prime}\left(u^{*}\right)$, i.e., $u^{*}=\bar{u}$. The latter means

$$
\begin{aligned}
\eta_{s}\left(u^{*}\right)= & \frac{g\left(u^{*}\right)-g\left(u_{3}\right)}{u^{*}-u_{3}}+\frac{f^{\prime}\left(u^{*}\right)-\left(f\left(u^{*}\right)-f\left(u_{3}\right)\right) /\left(u^{*}-u_{3}\right)}{f^{\prime}\left(u_{3}\right)-\left(f\left(u^{*}\right)-f\left(u_{3}\right)\right) /\left(u^{*}-u_{3}\right)} \\
& \times\left(g^{\prime}\left(u_{3}\right)-\frac{g\left(u^{*}\right)-g\left(u_{3}\right)}{u^{*}-u_{3}}\right) .
\end{aligned}
$$

According to inequality (B), we have

$$
\begin{aligned}
\eta_{s}(\bar{u})= & g^{\prime}(\bar{u})<\frac{g(\bar{u})-g\left(u_{3}\right)}{\bar{u}-u_{3}}+\frac{f^{\prime}(\bar{u})-\left(f(\bar{u})-f\left(u_{3}\right)\right) /\left(\bar{u}-u_{3}\right)}{f^{\prime}\left(u_{3}\right)-\left(f(\bar{u})-f\left(u_{3}\right)\right) /\left(\bar{u}-u_{3}\right)} \\
& \times\left(g^{\prime}\left(u_{3}\right)-\frac{g(\bar{u})-g\left(u_{3}\right)}{\bar{u}-u_{3}}\right)
\end{aligned}
$$

and when $u=u_{2}$,

$$
\eta_{s}\left(u_{2}\right)=g_{2,3}^{\prime}>g_{2,3}^{\prime}+\frac{f^{\prime}\left(u_{2}\right)-f_{2,3}^{\prime}}{f^{\prime}\left(u_{3}\right)-f_{2,3}^{\prime}}\left(g^{\prime}\left(u_{3}\right)-g_{2,3}^{\prime}\right)
$$

holds. Thus there exists a $u^{*} \in\left(u_{2}, \bar{u}\right)$ satisfying (12). So we need only consider the latter possibility of $u^{*} \in\left(u_{2}, \bar{u}\right)$ such that $\eta_{s}(u)>g^{\prime}(u)$ for $u \in\left[u_{2}, u^{*}\right]$, and therefore

$$
d^{2} \eta_{s} / d \xi^{2}>0 \text { for } u \in\left(u_{2}, u^{*}\right]
$$

For $u>u^{*}$ the shock continues as a half contact discontinuity which separates the original rarefaction wave from a new, compactly supported rarefaction wave which lies on the $u_{3}$ side of the discontinuity (see Figure 4.1). The discontinuity is a half contact discontinuity on the $u_{3}$ side because the tangency condition (12) is satisfied at every point $(\xi, \eta)$ on the discontinuity, with $u_{3}$ replaced by $u^{-}$and $u^{*}$ replaced by $u^{+}(\xi)=\left(f^{\prime}\right)^{-1}(\xi)$. Consequently $\eta=\eta_{c}(\xi)$ and $u^{-}$must satisfy (note that $u^{-}(\xi)$ is not required to be less than $u^{+}$)

$$
\left\{\begin{array}{l}
\frac{d \eta_{c}}{d \xi} \stackrel{(\mathrm{R}-\mathrm{H})}{=} \frac{\left(g\left(u^{+}\right)-g\left(u^{-}\right)\right) /\left(u^{+}-u^{-}\right)-\eta_{c}}{\left(f\left(u^{+}\right)-f\left(u^{-}\right)\right) /\left(u^{+}-u^{-}\right)-\xi} \\
\quad \stackrel{(\mathrm{E})}{=} \frac{g^{\prime}\left(u^{-}\right)-\left(g\left(u^{+}\right)-g\left(u^{-}\right)\right) /\left(u^{+}-u^{-}\right)}{f^{\prime}\left(u^{-}\right)-\left(f\left(u^{+}\right)-f\left(u^{-}\right)\right) /\left(u^{+}-u^{-}\right)} \\
\xi=f^{\prime}\left(u^{+}\right), \\
\left.\eta_{c}\right|_{\xi=f^{\prime}\left(u^{*}\right)}=\eta_{s}\left(u^{*}\right) .
\end{array}\right.
$$

We shall change the form of (13) and then prove the existence of $\eta$ and $u^{-}$ until the shock hits a point on the base curve. 
Differentiating (E) in (13), we get

$$
\begin{aligned}
& \frac{g^{\prime \prime}\left(u^{-}\right) \frac{d u^{-}}{d \xi}-\frac{\left(g^{\prime}\left(u^{+}\right) \frac{d u^{+}}{d \xi}-g^{\prime}\left(u^{-}\right) \frac{d u^{-}}{d \xi}\right)\left(u^{+}-u^{-}\right)-\left(g\left(u^{+}\right)-g\left(u^{-}\right)\right)\left(\frac{d u^{+}}{d \xi}-\frac{d u^{-}}{d \xi}\right)}{\left(u^{+}-u^{-}\right)^{2}}}{f^{\prime}\left(u^{-}\right)-\frac{f\left(u^{+}\right)-f\left(u^{-}\right)}{u^{+}-u^{-}}} \\
& -\frac{g^{\prime}\left(u^{-}\right)-\frac{g\left(\dot{u}^{+}\right)-g\left(u^{-}\right)}{u^{+}-u^{-}}}{\left(f^{\prime}\left(u^{-}\right)-\frac{f\left(u^{+}\right)-f\left(u^{-}\right)}{u^{+}-u^{-}}\right)^{2}}\left[f^{\prime \prime}\left(u^{-}\right) \frac{d u^{-}}{d \xi}-\right. \\
& \left.\frac{\left(f^{\prime}\left(u^{+}\right) \frac{d u^{+}}{d \xi}-f^{\prime}\left(u^{-}\right) \frac{d u^{-}}{d \xi}\right)\left(u^{+}-u^{-}\right)-\left(f\left(u^{+}\right)-f\left(u^{-}\right)\right)\left(\frac{d u^{+}}{d \xi}-\frac{d u^{-}}{d \xi}\right)}{\left(u^{+}-u^{-}\right)^{2}}\right] \\
& =\frac{\frac{\left(g^{\prime}\left(u^{+}\right) \frac{d u^{+}}{d \xi}-g^{\prime}\left(u^{-}\right) \frac{d u^{-}}{d \xi}\right)\left(u^{+}-u^{-}\right)-\left(g\left(u^{+}\right)-g\left(u^{-}\right)\right)\left(\frac{d u^{+}}{d \xi}-\frac{d u^{-}}{d \xi}\right)}{\left(u^{+}-u^{-}\right)^{2}}-\frac{d \eta_{c}(\xi)}{d \xi}}{\frac{f\left(u^{+}\right)-f\left(u^{-}\right)}{u^{+}-u^{-}}-f^{\prime}\left(u^{+}\right)} \\
& +\frac{\frac{g\left(u^{+}\right)-g\left(u^{-}\right)}{u^{+}-u^{-}}-\eta_{c}(\xi)}{\left(\frac{f\left(u^{+}\right)-f\left(u^{-}\right)}{u^{+}-u^{-}}-f^{\prime}\left(u^{+}\right)\right)^{2}}[1- \\
& \left.\frac{\left(f^{\prime}\left(u^{+}\right) \frac{d u^{+}}{d \xi}-f^{\prime}\left(u^{-}\right) \frac{d u^{-}}{d \xi}\right)\left(u^{+}-u^{-}\right)-\left(f\left(u^{+}\right)-f\left(u^{-}\right)\right)\left(\frac{d u^{+}}{d \xi}-\frac{d u^{-}}{d \xi}\right)}{\left(u^{+}-u^{-}\right)^{2}}\right] \text {. }
\end{aligned}
$$

After some calculations, we have

$$
\begin{aligned}
\frac{d u^{-}}{d \xi}= & \frac{1}{f^{\prime \prime}\left(u^{+}\right) g^{\prime \prime}\left(u^{-}\right)} \cdot \frac{f^{\prime}\left(u^{-}\right)-f^{\prime}\left(u^{+}\right)}{u^{+}-u^{-}} \cdot \frac{f^{\prime}\left(u^{-}\right)-f_{+,-}^{\prime}}{g^{\prime}\left(u^{-}\right)-g_{+,-}^{\prime}} \\
& \cdot\left(\frac{g^{\prime}\left(u^{-}\right)-g_{+,-}^{\prime}}{f^{\prime}\left(u^{-}\right)-f_{+,-}^{\prime}}-\frac{g^{\prime}\left(u^{+}\right)-g_{+,-}^{\prime}}{f^{\prime}\left(u^{+}\right)-f_{+,-}^{\prime}}\right) /\left(\frac{f^{\prime}\left(u^{-}\right)-f_{+,-}^{\prime}}{g^{\prime}\left(u^{-}\right)-g_{+,-}^{\prime}}-\frac{f^{\prime \prime}\left(u^{-}\right)}{g^{\prime \prime}\left(u^{-}\right)}\right) .
\end{aligned}
$$

This equation combined with the first equation in (13) gives the existence of $\eta=\eta_{c}(\xi)$ and $u^{-}=u^{-}(\xi)$ with initial data $\left(u^{+}, u^{-}\right)=\left(u^{*}, u_{3}\right)\left(u^{*}<u_{3}\right)$ until a point $u^{+}=u^{-}$(denoted by $\tilde{u}$ ) or $u^{+}=u_{3}$. When $u^{-}>u^{+}$, i.e., $u^{+}<\tilde{u}$, we know that $d \eta_{c} / d \xi>0$ from (E) in (13), and from inequalities (A) and (B), we know that $d u^{-} / d \xi<0$. Moreover,

$$
\left.\frac{d \eta_{c}}{d \xi}\right|_{u=\tilde{u}}=\lim _{u^{-}, u^{+} \rightarrow \tilde{u}} \frac{d \eta_{c}}{d \xi}=\lim _{u^{+}, u^{-} \rightarrow \tilde{u}} \frac{g^{\prime}\left(u^{-}\right)-g_{+,-}^{\prime}}{f^{\prime}\left(u^{-}\right)-f_{+,-}^{\prime}}=\frac{g^{\prime \prime}(\tilde{u})}{f^{\prime \prime}(\tilde{u})} .
$$

Thus, from (E) in (13),

$$
\lim _{u^{+} \rightarrow \tilde{u}} \frac{g_{+,-}^{\prime}-\eta_{c}}{f_{+,-}^{\prime}-f_{+}^{\prime}}=\frac{g^{\prime \prime}(\tilde{u})}{f^{\prime \prime}(\tilde{u})}
$$

This gives $\eta_{c}\left(u^{-}\right)=g^{\prime}\left(u^{-}\right)$; i.e., the half contact discontinuity line $\eta=\eta_{c}(\xi)$ ends at a point on the base curve and is tangent to the base curve at this point. Because $u^{-}$decreases from $u_{3}$ with increasing $u^{+}$, we know that $\tilde{u}=u^{+}=$ $u^{-}<u_{3}$. 
From (13), we have

$$
\frac{d \eta_{c}}{d \xi}=\frac{g^{\prime}\left(u^{-}\right)-\eta_{c}}{f^{\prime}\left(u^{-}\right)-\xi}
$$

This means that the tangent line of $\eta=\eta_{c}(u)$ at $u=u^{+}$is just the characteristic line generating from the point $\left(f^{\prime}\left(u^{-}\right), g^{\prime}\left(u^{-}\right)\right)$on the base curve.

We show that $\eta=\eta_{c}(\xi)$ is convex as follows:

$$
\begin{aligned}
\frac{d^{2} \eta_{c}}{d \xi^{2}}= & \frac{1}{f^{\prime \prime}\left(u^{+}\right)}\left(\frac{\partial}{\partial u^{+}}\left(\frac{d \eta_{c}}{d \xi}\right)\right)+\frac{\partial}{\partial u^{-}}\left(\frac{d \eta_{c}}{d \xi}\right) \frac{d u^{-}}{d \xi} \\
= & \frac{\left(\frac{-g^{\prime}\left(u^{+}\right)}{u^{+}-u^{-}}+\frac{g\left(u^{+}\right)-g\left(u^{-}\right)}{\left(u^{+}-u^{-}\right)^{2}}\right)\left(f^{\prime}\left(u^{-}\right)-f_{+,-}^{\prime}\right)}{f^{\prime \prime}\left(u^{+}\right)\left(f^{\prime}\left(u^{-}\right)-f_{+,-}^{\prime}\right)^{2}} \\
& -\frac{\left(g^{\prime}\left(u^{-}\right)-g_{+,-}^{\prime}\right)\left(\frac{-f^{\prime}\left(u^{+}\right)}{u^{+}-u^{-}}+\frac{f\left(u^{+}\right)-f\left(u^{-}\right)}{\left(u^{+}-u^{-}\right)^{2}}\right)}{f^{\prime \prime}\left(u^{+}\right)\left(f^{\prime}\left(u^{-}\right)-f_{+,-}^{\prime}\right)^{2}} \\
& +\frac{d u^{-}}{d \xi} \cdot \frac{g^{\prime \prime}\left(u^{-}\right)+\frac{g^{\prime}\left(u^{-}\right)}{u^{+}-u^{-}}-\frac{g\left(u^{+}\right)-g\left(u^{-}\right)}{\left(u^{+}-u^{-}\right)^{2}}}{f^{\prime}\left(u^{-}\right)-f_{+,-}^{\prime}} \\
& -\frac{d u^{-}\left(g^{\prime}\left(u^{-}\right)-g_{+,-}^{\prime}\right)\left(f^{\prime \prime}\left(u^{-}\right)-\frac{f^{\prime}\left(u^{-}\right)}{u^{+}-u^{-}}-\frac{f\left(u^{+}\right)-f\left(u^{-}\right)}{\left(u^{+}-u^{-}\right)^{2}}\right)}{\left(f^{\prime}\left(u^{-}\right)-f_{+,-}^{\prime}\right)^{2}} \\
= & -\frac{1}{\left(u^{+}-u^{-}\right) f^{\prime \prime}\left(u^{+}\right)}\left(\frac{g^{\prime}\left(u^{+}\right)-g_{+,-}^{\prime}}{f^{\prime}\left(u^{+}\right)-f_{+,-}^{\prime}} \frac{g^{\prime}\left(u^{-}\right)-g_{+,-}^{\prime}}{f^{\prime}\left(u^{-}\right)-f_{+,-}^{\prime}}\right)>0 .
\end{aligned}
$$

We have shown that the solution for this case has the properties depicted in Figure 4.1, case $\left(c_{2}\right)$.

\section{CASE (d): exactly ONE RAREFACTION WAVe}

In this case, there are two possible orderings of initial data: $u_{1} \leq u_{2} \leq u_{3}<$ $u_{4}$ or $u_{2}<u_{1} \leq u_{4} \leq u_{3}$. For the former ordering, we construct the solution as in Figure 5.1. The shock $\eta=g_{1.4}^{\prime}$ penetrates the planar rarefaction wave $\xi=f^{\prime}(u), u_{3} \leq u \leq u_{4}$ similar to case $\left(\mathrm{c}_{1}\right)$. At the end-point, the slope of the tangent line is

$$
\frac{d \eta}{d \xi}=\frac{g_{1,3}^{\prime}-\eta}{f_{1,3}^{\prime}-f^{\prime}\left(u_{3}\right)} .
$$

(We assume here that $u_{1} \neq u_{3}$ since we have considered the case $u_{1}=u_{2}=$ $u_{3}<u_{4}$ in the first part of case $\left(c_{1}\right)$.) Therefore, it passes through point $\left(f_{1,3}^{\prime}, g_{1,3}^{\prime}\right)$ and satisfies the $\mathbf{R}-\mathrm{H}$ relation and the entropy condition as well. The other discontinuity lines are obviously admissible.

There are quite different configurations of solutions to the other ordering of initial data. The simplest case is $\left(\mathrm{d}_{2}\right): u_{2}<u_{1} \leq u^{*}\left(u_{2}, u_{3}\right) \leq u_{4} \leq u_{3}$. The shock $\eta=g_{2,3}^{\prime}$ penetrates the rarefaction wave $\xi=f^{\prime}(u), u_{2} \leq u \leq u_{1}$ and the solution can be constructed as in case $\left(d_{2}\right)$ (Figure 5.2). 


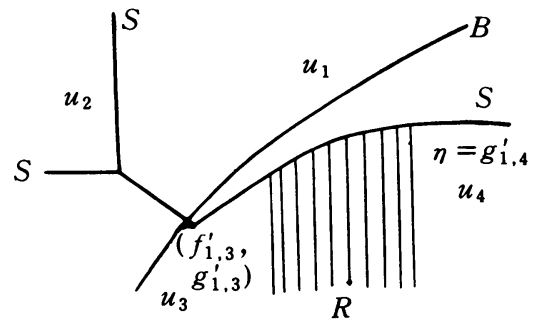

case $\left(\mathrm{d}_{1}\right): u_{1} \leq u_{2} \leq u_{3}<u_{4}$

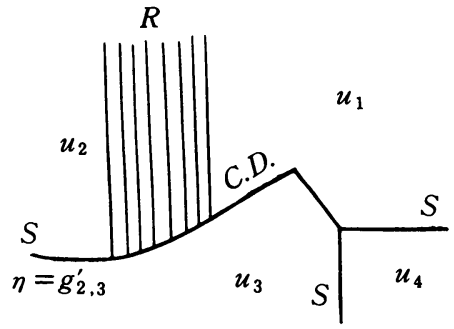

case $\left(\mathrm{d}_{2}\right): u_{2}<u_{1} \leq u^{*}\left(u_{2}, u_{3}\right) \leq u_{4} \leq u_{3}$

FIGURE 5.1

FIGURE 5.2

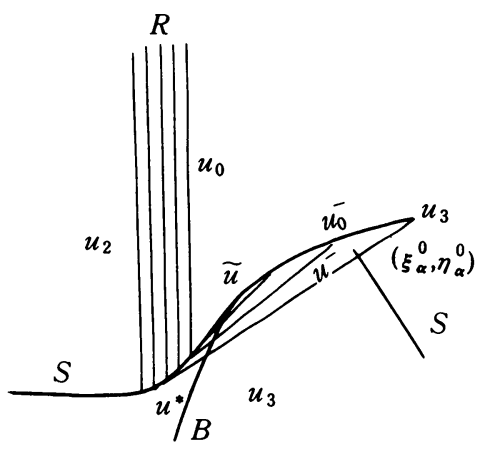

FIGURE 5.3

The solutions for the other cases contain envelope rarefaction waves similar to the one which occurs in case $\left(c_{2}\right)$. These waves differ from the previous case in that the characteristics terminate in discontinuities at both ends. At one end we have a half contact discontinuity, $\eta=\eta_{c}(\xi)$, as in case $\left(c_{2}\right)$. At the other end we have a shock wave, with $u=u^{-}(\xi)$ on one side, and $u=u_{1}$ (or $u_{0}$ ) on the other. This new shock curve $\eta=\eta(\xi)$ (or $\eta=\eta_{d}(\xi)$ ) satisfies

$$
\frac{d \eta}{d \xi}=\left(\frac{g\left(u^{-}(\xi, \eta)\right)-g\left(u_{0}\right)}{u^{-}(\xi, \eta)-u_{0}}-\eta\right) /\left(\frac{f\left(u^{-}(\xi, \eta)\right)-f\left(u_{0}\right)}{u^{-}(\xi, \eta)-u_{0}}-\xi\right),
$$

where $u^{-}(\xi, \eta)=u^{-}$satisfies

$$
\begin{aligned}
& \eta-\eta_{c}\left(u^{-}\right)=\frac{g^{\prime}\left(u^{-}\right)-g_{+,-}^{\prime}}{f^{\prime}\left(u^{-}\right)-f_{+,-}^{\prime}}\left(\xi-f^{\prime}\left(u^{+}\right)\right), \\
& \eta-\eta_{c}\left(u^{-}\right)=\frac{g^{\prime}\left(u^{-}\right)-\eta_{c}\left(u^{-}\right)}{f^{\prime}\left(u^{-}\right)-f^{\prime}\left(u^{+}\right)}\left(\xi-f^{\prime}\left(u^{+}\right)\right), \\
& \eta-g^{\prime}\left(u^{-}\right)=\frac{g^{\prime}\left(u^{-}\right)-g_{+,-}^{\prime}}{f^{\prime}\left(u^{-}\right)-f_{+,-}^{\prime}}\left(\xi-f^{\prime}\left(u^{-}\right)\right),
\end{aligned}
$$

with

$$
\eta_{c}\left(u^{-}\right)=g_{+,-}^{\prime}+\left(f^{\prime}\left(u^{+}\right)-f_{+,-}^{\prime}\right)\left(g^{\prime}\left(u^{-}\right)-g_{+,-}^{\prime}\right) /\left(f^{\prime}\left(u^{-}\right)-f_{+,-}^{\prime}\right),
$$

$u^{+}=u^{+}\left(u^{-}\right)$, and $u^{*}\left(u_{2}, u_{3}\right)<u_{0}<u_{3} ; u^{*}$ and $\tilde{u}$ are both determined by $u_{2}$ and $u_{3}$ (Figure 5.3). 
We start by considering the general properties of integral curves of equation (14). The singular point of equation (14) can be obtained from (15.3) and

$$
\xi=\frac{f\left(u^{-}\right)-f\left(u_{0}\right)}{u^{-}-u_{0}}, \quad \eta=\frac{g\left(u^{-}\right)-g\left(u_{0}\right)}{u^{-}-u_{0}}
$$

This is equivalent to obtaining $u^{-}$from

$$
\frac{g^{\prime}\left(u^{-}\right)-g_{+,-}^{\prime}}{f^{\prime}\left(u^{-}\right)-f_{+,-}^{\prime}}=\frac{g^{\prime}\left(u^{-}\right)-g_{-, 0}^{\prime}}{f^{\prime}\left(u^{-}\right)-f_{-, 0}^{\prime}}
$$

We prove an inequality:

$$
\frac{g^{\prime}\left(u^{-}\right)-g_{+,-}^{\prime}}{f^{\prime}\left(u^{-}\right)-f_{+,-}^{\prime}}>\frac{g^{\prime}\left(u^{-}\right)-g_{-, 0}^{\prime}}{f^{\prime}\left(u^{-}\right)-f_{-, 0}^{\prime}}, \text { for } u^{+}<u_{0}<u^{-}
$$

Let

$$
F\left(u^{+}, u^{-}\right)=\frac{g^{\prime}\left(u^{-}\right)-g_{+,-}^{\prime}}{f^{\prime}\left(u^{-}\right)-f_{+,-}^{\prime}}
$$

then

$$
\begin{aligned}
\frac{\partial F\left(u^{+}, u^{-}\right)}{\partial u^{+}}= & \frac{1}{u^{+}-u^{-}} \cdot \frac{f^{\prime}\left(u^{+}\right)-f_{+,-}^{\prime}}{f^{\prime}\left(u^{-}\right)-f_{+,-}^{\prime}} \\
& \times\left(\frac{g^{\prime}\left(u^{-}\right)-g_{+,-}^{\prime}}{f^{\prime}\left(u^{-}\right)-f_{+,-}^{\prime}}-\frac{g^{\prime}\left(u^{+}\right)-g_{+,-}^{\prime}}{f^{\prime}\left(u^{+}\right)-f_{+,-}^{\prime}}\right)<0, \quad u^{+}<u^{-} ;
\end{aligned}
$$

i.e., $F\left(u^{+}, u^{-}\right)$is a monotonically decreasing function of $u^{+}$for $u^{+}<u^{-}$. Thus inequality $(C)$ follows. Note that $(C)$ is equivalent to the inequality

$$
\frac{g\left(u^{-}\right)-g\left(u_{0}\right)}{u^{-}-u_{0}}>\eta_{c}\left(u^{-}\right)+\frac{g^{\prime}\left(u^{-}\right)-g_{+,-}^{\prime}}{f^{\prime}\left(u^{-}\right)-f_{+,-}^{\prime}}\left(\frac{f\left(u^{-}\right)-f\left(u_{0}\right)}{u^{-}-u_{0}}-f^{\prime}\left(u^{+}\right)\right)
$$

for any $u^{+}<u_{0}<u^{-} \leq u_{3}$. We will use (16) later.

Thus, the singular points of equation (14) are either $\xi=f^{\prime}\left(u_{0}\right), \eta=g^{\prime}\left(u_{0}\right)$, or

$$
\xi=\frac{f\left(u_{0}^{-}\right)-f\left(u_{0}\right)}{u_{0}^{-}-u_{0}}, \quad \eta=\frac{g\left(u_{0}^{-}\right)-g\left(u_{0}\right)}{u_{0}^{-}-u_{0}}
$$

where $u_{0}^{-}$denotes the corresponding point of $u^{+}=u_{0}<\tilde{u}$. 
Assume $\eta=\eta_{d}(\xi)$ is a solution to equation (14), with $u^{-}$the value of $u$ on the inner side $u^{-}=u^{-}(\xi)=u^{-}\left(\xi, \eta_{d}(\xi)\right)$. Then, we differentiate (15.2) to get

$$
\begin{aligned}
\frac{d \eta_{d}}{d \xi}- & \frac{d \eta_{c}\left(u^{-}\right)}{d u^{-}} \cdot \frac{d u^{-}}{d \xi} \\
= & \frac{\xi-f^{\prime}\left(u^{+}\right)}{f^{\prime}\left(u^{-}\right)-f^{\prime}\left(u^{+}\right)}\left(g^{\prime \prime}\left(u^{-}\right) \frac{d u^{-}}{d \xi}-\frac{d \eta_{c}\left(u^{-}\right)}{d u^{-}} \frac{d u^{-}}{d \xi}\right) \\
& +\frac{g^{\prime}\left(u^{-}\right)-\eta_{c}\left(u^{-}\right)}{f^{\prime}\left(u^{-}\right)-f^{\prime}\left(u^{+}\right)}\left(1-f^{\prime \prime}\left(u^{+}\right) \frac{d u^{+}}{d u^{-}} \frac{d u^{-}}{d \xi}\right) \\
& -\frac{g^{\prime}\left(u^{-}\right)-\eta_{c}\left(u^{-}\right)}{\left(f^{\prime}\left(u^{-}\right)-f^{\prime}\left(u^{+}\right)\right)^{2}}\left(\xi-f^{\prime}\left(u^{+}\right)\right)\left(f^{\prime \prime}\left(u^{-}\right)-f^{\prime \prime}\left(u^{+}\right) \frac{d u^{+}}{d u^{-}}\right) \frac{d u^{-}}{d \xi}
\end{aligned}
$$

Making use of

$$
\frac{d \eta_{c}\left(u^{-}\right)}{d u^{-}}=\frac{d \eta_{c}}{d \xi} \cdot \frac{d \xi}{d u^{+}} \cdot \frac{d u^{+}}{d u^{-}}=\frac{g^{\prime}\left(u^{-}\right)-\eta_{c}\left(u^{-}\right)}{f^{\prime}\left(u^{-}\right)-f^{\prime}\left(u^{+}\right)} \cdot f^{\prime \prime}\left(u^{+}\right) \cdot \frac{d u^{+}}{d u^{-}}
$$

we have

$$
\frac{d u^{-}}{d \xi}=\frac{\frac{d \eta_{d}}{d \xi}-\frac{g^{\prime}\left(u^{-}\right)-\eta_{c}\left(u^{-}\right)}{f^{\prime}\left(u^{-}\right)-f^{\prime}\left(u^{+}\right)}}{\left(\eta-\eta_{c}\left(u^{-}\right)\right)\left(\frac{g^{\prime \prime}\left(u^{-}\right)}{g^{\prime}\left(u^{-}\right)-\eta_{c}\left(u^{-}\right)}-\frac{f^{\prime \prime}\left(u^{-}\right)}{f^{\prime}\left(u^{-}\right)-f^{\prime}\left(u^{+}\right)}\right)}
$$

and

$$
\frac{d^{2} \eta_{d}}{d \xi^{2}}=\frac{1}{u^{-}-u_{0}} \frac{f^{\prime}\left(u^{-}\right)-\frac{f^{\prime}\left(u^{-}\right)-f\left(u_{0}\right)}{u^{-}-u_{0}}}{\frac{f\left(u^{-}\right)-f\left(u_{0}\right)}{u^{-}-u_{0}}-\xi}\left(\frac{g^{\prime}\left(u^{-}\right)-g_{-, 0}^{\prime}}{f^{\prime}\left(u^{-}\right)-f_{-, 0}^{\prime}}-\frac{d \eta_{d}}{d \xi}\right) \frac{d u^{-}}{d \xi}
$$

By contrast, if we assume $\xi=\xi_{d}(\eta)$ is a solution, $u^{-}=u^{-}\left(\xi_{d}(\eta), \eta\right)$, we have analogously

$$
\begin{gathered}
\frac{d u^{-}}{d \eta}=\frac{1-\frac{d \xi_{d}}{d \eta} \cdot \frac{g^{\prime}\left(u^{-}\right)-\eta_{c}\left(u^{-}\right)}{f^{\prime}\left(u^{-}\right)-f^{\prime}\left(u^{+}\right)}}{\left(\eta-\eta_{c}\left(u^{-}\right)\right)\left(\frac{g^{\prime \prime}\left(u^{-}\right)}{g^{\prime}\left(u^{-}\right)-\eta_{c}\left(u^{-}\right)}-\frac{f^{\prime \prime}\left(u^{-}\right)}{f^{\prime}\left(u^{-}\right)-f^{\prime}\left(u^{+}\right)}\right)}, \\
\frac{d^{2} \xi_{d}}{d \eta^{2}}=\frac{1}{u^{-}-u_{0}} \cdot \frac{g^{\prime}\left(u^{-}\right)-g_{-, 0}^{\prime}}{g_{-, 0}^{\prime}-\eta}\left(\frac{f^{\prime}\left(u^{-}\right)-f_{-, 0}^{\prime}}{g^{\prime}\left(u^{-}\right)-g_{-, 0}^{\prime}}-\frac{d \xi_{d}}{d \eta}\right) \frac{d u^{-}}{d \eta} .
\end{gathered}
$$

Suppose, on the integral curve $\eta=\eta_{d}(\xi)$, there is a point $u^{-}$at which the discontinuity in $u$ is a half contact discontinuity on the $u^{-}$side. Then, as in (11), we must have

$$
\frac{d \eta_{d}}{d \xi}=\frac{g_{-, 0}^{\prime}-\eta_{d}}{f_{-, 0}^{\prime}-\xi}=\frac{g^{\prime}\left(u^{-}\right)-g_{-, 0}^{\prime}}{f^{\prime}\left(u^{-}\right)-f_{-, 0}^{\prime}}=\frac{g^{\prime}\left(u^{-}\right)-\eta_{d}}{f^{\prime}\left(u^{-}\right)-\xi}
$$

Making use of (15.3), we have

$$
\frac{g^{\prime}\left(u^{-}\right)-g_{-, 0}^{\prime}}{f^{\prime}\left(u^{-}\right)-f_{-, 0}^{\prime}}=\frac{g^{\prime}\left(u^{-}\right)-g_{+,-}^{\prime}}{f^{\prime}\left(u^{-}\right)-f_{+,-}^{\prime}}
$$




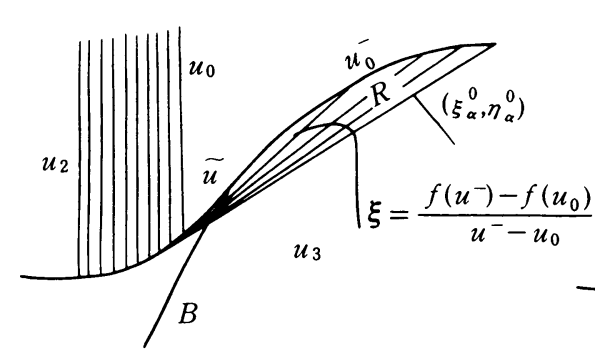

$$
u_{0}<\tilde{u}
$$

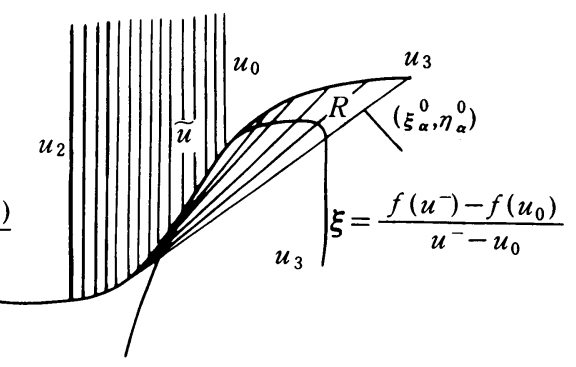

$$
u_{0}>\tilde{u}
$$

\section{FIGURE 5.4}

Consequently $u^{+}=u_{0}$ or $u^{-}=u_{0}$ due to inequality (C).

We remark that it is obvious to deduce $u^{+}=u_{0}$ or $u^{-}=u_{0}$ under the assumption

$$
\frac{d \eta_{d}}{d \xi}=\frac{g^{\prime}\left(u^{-}\right)-g_{-, 0}^{\prime}}{f^{\prime}\left(u^{-}\right)-f_{-, 0}^{\prime}}
$$

We shall consider equation (14) with different initial data in order to get solutions we need later.

$1^{\circ}$. Let $\left(\xi_{\alpha}^{0}, \eta_{\alpha}^{0}\right)$ be a point on the characteristic $u^{-}=u_{3}$ connecting $\left(f^{\prime}\left(u^{*}\right), \eta_{s}\left(u^{*}\right)\right)$ and $\left(f^{\prime}\left(u_{3}\right), g^{\prime}\left(u_{3}\right)\right)$ such that $f^{\prime}\left(u_{3}\right)>\xi_{\alpha}^{0}>f_{3,0}^{\prime}$, and let

$$
\alpha=\frac{g_{3,0}^{\prime}-\eta_{\alpha}^{0}}{f_{3,0}^{\prime}-\xi_{\alpha}^{0}}
$$

Let $\eta_{\alpha}(\xi)$ be the integral curve of (14) through $\left(\xi_{\alpha}^{0}, \eta_{\alpha}^{0}\right)$. Then

$$
\left.\frac{d \eta_{\alpha}}{d \xi}\right|_{\left(\xi_{*}^{0}, \eta_{t}^{0}\right)}=\alpha<\frac{g^{\prime}\left(u_{3}\right)-\eta_{s}\left(u^{*}\right)}{f^{\prime}\left(u_{3}\right)-f^{\prime}\left(u^{*}\right)}
$$

Since the right-hand side of this inequality is the slope of the characteristic $u^{-}=u_{3}$, one branch of the integral curve must enter the region of the envelope rarefaction wave (see Figure 5.4). We shall prove that the integral curves will not leave the region $R$ (cf. Figure 5.4):

$$
\begin{aligned}
& R \equiv\{(\xi, \eta) \mid(\xi, \eta) \text { exists between the four curves } \\
& \qquad \begin{aligned}
\xi=f^{\prime}(u), \eta=g^{\prime}(u), \text { and } \xi=\frac{f\left(u^{-}(\xi, \eta)\right)-f\left(u_{0}\right)}{u^{-}(\xi, \eta)-u_{0}}, \text { and } \\
\left.u^{-}(\xi, \eta)=u_{3}, \text { and } u^{-}(\xi, \eta)=u_{0}^{-}, u_{0}\left(u_{0}^{-}\right)<u^{-}<u_{3}\right\}
\end{aligned}
\end{aligned}
$$

except at the singular points

$$
\left(f^{\prime}\left(u_{0}\right), g^{\prime}\left(u_{0}\right)\right) \text { or }\left(\frac{f\left(u_{0}^{-}\right)-f\left(u_{0}\right)}{u_{0}^{-}-u_{0}}, \frac{g\left(u_{0}^{-}\right)-g\left(u_{0}\right)}{u_{0}^{-}-u_{0}}\right) .
$$


As

$$
\left.\frac{d \eta}{d \xi}\right|_{\left(\xi_{, !}^{0}, \eta_{n}^{0}\right)}=\alpha<\frac{g^{\prime}\left(u_{3}\right)-g_{3,0}^{\prime}}{f^{\prime}\left(u_{3}\right)-f_{3,0}^{\prime}} \text { and } \frac{f\left(u_{3}\right)-f\left(u_{0}\right)}{u_{3}-u_{0}}<\xi_{\alpha}^{0},
$$

before the integral curves leave $R$, there must hold

$$
\frac{f\left(u^{-}\left(\xi, \eta_{\alpha}\right)\right)-f\left(u_{0}\right)}{u^{-}\left(\xi, \eta_{\alpha}\right)-u_{0}}<\xi,
$$

and

$$
\frac{d \eta_{\alpha}}{d \xi}<\frac{g^{\prime}\left(u^{-}\left(\xi, \eta_{\alpha}(\xi)\right)\right)-g_{-, 0}^{\prime}}{f^{\prime}\left(u^{-}\left(\xi, \eta_{\alpha}(\xi)\right)\right)-f_{-, 0}^{\prime}}, \quad u^{-}=u^{-}\left(\xi, \eta_{\alpha}(\xi)\right)
$$

in view of the previous remark. Therefore,

$$
\frac{d^{2} \eta_{\alpha}}{d \xi^{2}}<0 \quad \text { or } \quad \alpha \leq \frac{d \eta_{\alpha}}{d \xi}<\frac{g^{\prime}\left(u^{-}\left(\xi, \eta_{\alpha}(\xi)\right)\right)-g_{-, 0}^{\prime}}{f^{\prime}\left(u^{-}\left(\xi, \eta_{\alpha}(\xi)\right)\right)-f_{-, 0}^{\prime}}
$$

Hence, when the integral curves intersect the curve

$$
\xi=\frac{f\left(u^{-}(\xi, \eta)\right)-f\left(u_{0}\right)}{u^{-}(\xi, \eta)-u_{0}}
$$

it must be that

$$
\eta=\frac{g\left(u^{-}(\xi, \eta)\right)-g\left(u_{0}\right)}{u^{-}(\xi, \eta)-u_{0}}
$$

simultaneously. Thus from (C) and (13), we have $\xi=f^{\prime}\left(u_{0}\right), \eta=g^{\prime}\left(u_{0}\right)$ or

$$
\xi=\frac{f\left(u_{0}^{-}\right)-f\left(u_{0}\right)}{u_{0}^{-}-u_{0}}, \quad \eta=\frac{g\left(u_{0}^{-}\right)-g\left(u_{0}\right)}{u_{0}^{-}-u_{0}} .
$$

Similarly as in case $\left(c_{1}\right)$, we can prove that the integral curves cannot intersect the base curve (using $(\mathrm{A})$ ). It is easy to show that the integral curves will not go out through the line $u^{-}=u_{3}$ using the geometric theory of ordinary differential equations. Also when $u_{0}<\tilde{u}, u^{-}(\xi, \eta)=u_{0}^{-}$is an integral curve; thus, the integral curves must end at the singular point. Thus we have proved what we claimed and

$$
\frac{d \eta_{\alpha}}{d \xi} \geq \alpha, \quad \frac{d^{2} \eta_{\alpha}}{d \xi^{2}}<0, \quad \text { and } \quad \frac{d u^{-}\left(\xi, \eta_{\alpha}(\xi)\right)}{d \xi}>0
$$

We next prove that this solution satisfies the entropy condition (10). Suppose the normal to the discontinuity is $\vec{n}=(\alpha, \beta)$ pointing from $u_{0}$ to $u^{-}$. If $\alpha \leq 0$ and $\beta \leq 0$, then $F=\alpha f+\beta g$ is concave and thus the entropy condition is satisfied. For the other cases, we need only prove that the entropy condition (10) is not violated because of a half contact discontinuity on the side of the integral curve which faces the region of constant state $u_{0}$. In fact, we have $\alpha>0, \beta<0,\left(g^{\prime \prime} / f^{\prime \prime}\right)(\stackrel{\circ}{u})=\alpha /(-\beta)$, and $\stackrel{\circ}{u}(\alpha /-\beta)$ decreases from $+\infty$ 
with $\xi$ decreasing from $\xi_{\alpha}^{0}$ or some value less than $\xi_{\alpha}^{0}$. This is enough to ensure what we want to prove.

$2^{\circ}$. Suppose $\eta=\eta_{\alpha}(\xi)$ is an integral curve through the point $\left(\xi_{\alpha}^{0}, \eta_{\alpha}^{0}\right)$ satisfying

$$
\begin{aligned}
f^{\prime}\left(u_{0}\right) & <\xi_{\alpha}^{0}=f^{\prime}\left(u^{*}\right)+\frac{f^{\prime}\left(u_{3}\right)-f^{\prime}\left(u^{*}\right)}{g^{\prime}\left(u_{3}\right)-\eta_{s}\left(u_{3}\right)}\left(\eta_{\alpha}^{0}-\eta_{s}\left(u_{3}\right)\right) \\
& \leq \frac{f\left(u_{3}\right)-f\left(u_{0}\right)}{u_{3}-u_{0}}
\end{aligned}
$$

Then,

Therefore,

$$
\left.\frac{d \eta_{\alpha}}{d \xi}\right|_{\left(\xi_{n}^{0}, \eta_{n}^{0}\right)}=\alpha>\frac{g^{\prime}\left(u_{3}\right)-\eta_{s}\left(u_{3}\right)}{f^{\prime}\left(u_{3}\right)-f^{\prime}\left(u^{*}\right)} \stackrel{(\mathrm{C})}{>} \frac{g^{\prime}\left(u_{3}\right)-g_{3,0}^{\prime}}{f^{\prime}\left(u_{3}\right)-f_{3,0}^{\prime}}
$$

We know that if

$$
\left.\frac{d u^{-}}{d \xi}\right|_{\xi_{n}^{0}}<0,\left.\quad \frac{d^{2} \eta_{\alpha}}{d \xi^{2}}\right|_{\xi_{n}^{0}}>0
$$

$$
\frac{d \eta_{\alpha}}{d \xi}=\frac{g^{\prime}\left(u^{-}\left(\xi, \eta_{\alpha}(\xi)\right)\right)-\eta_{c}\left(u^{-}\right)}{f^{\prime}\left(u^{-}\right)-f^{\prime}\left(u^{+}\right)},
$$

then from (15.2), (15.3) and (14)

$$
\frac{d \eta_{\alpha}}{d \xi}=\frac{g^{\prime}\left(u^{-}\right)-g_{-, 0}^{\prime}}{f^{\prime}\left(u^{-}\right)-f_{-, 0}^{\prime}}
$$

holds. This will imply $u^{-}=u_{0}$ or $u^{+}=u_{0}$. Therefore if only $\eta=\eta_{\alpha}(\xi)$ is in the region of envelope rarefaction wave, we have

$$
\frac{d \eta_{\alpha}}{d \xi}>\frac{g^{\prime}\left(u^{-}\right)-\eta_{c}\left(u^{-}\right)}{f^{\prime}\left(u^{-}\right)-f^{\prime}\left(u^{+}\left(u^{-}\right)\right)}
$$

We have $d^{2} \eta_{\alpha} / d \xi^{2}>0$ when

$$
\xi<\frac{f\left(u^{-}(\xi, \eta)\right)-f\left(u_{0}\right)}{u^{-}(\xi, \eta)-u_{0}}
$$

and $\eta_{\alpha}$ will not meet the line $u^{-}(\xi, \eta)=u_{3}$ again. If the curve

$$
\xi=\frac{f\left(u^{-}(\xi, \eta)\right)-f\left(u_{0}\right)}{u^{-}(\xi, \eta)-u_{0}}
$$

intersects the integral curve $\eta_{\alpha}$ at a point $A\left(\xi_{A}, \eta_{\alpha}\left(\xi_{A}\right)\right)$, we let the corresponding $u^{-}$be denoted by $u_{A}^{-}$. We then change $\eta_{\alpha}(\xi)$ to $\xi=\xi_{\alpha}(\eta)$ to consider

$$
\frac{d \xi_{\alpha}}{d \eta}=\left(\frac{f\left(u^{-}\right)-f\left(u_{0}\right)}{u^{-}-u_{0}}-\xi\right) /\left(\frac{g\left(u^{-}\right)-g\left(u_{0}\right)}{u^{-}-u_{0}}-\eta\right),\left.\quad \xi_{\alpha}\right|_{\eta_{u}\left(\xi_{A}\right)}=\xi_{A} .
$$


At a neighbourhood of point $A$, we have

$$
\begin{gathered}
\frac{g\left(u_{A}^{-}\right)-g\left(u_{0}\right)}{u_{A}^{-}-u_{0}}>\eta_{A}, \quad \xi_{A}=\frac{f\left(u_{A}^{-}\right)-f\left(u_{0}\right)}{u_{A}^{-}-u_{0}} \\
\left.\frac{d \xi_{\alpha}}{d \eta}\right|_{A}=0,\left.\quad \frac{d u^{-}}{d \eta}\right|_{A}<0,\left.\quad \frac{d^{2} \xi_{\alpha}}{d \eta^{2}}\right|_{A}<0 .
\end{gathered}
$$

Therefore, the integral curve $\eta=\eta_{\alpha}(\xi)$ can be extended further along $\eta$-axis. After passing through $A, d \eta_{\alpha}(\xi) / d \xi<0$. Similarly as in case $1^{\circ}$, we can prove that for the integral curves, $d^{2} \eta_{\alpha} / d \xi^{2}<0$ and $d u^{-} / d \xi>0$, and the integral curves are bounded by the base curve from above. It is obvious that the integral curves cannot enter into the region $\xi<f^{\prime}\left(u_{0}\right)$. Thus the solutions must end at the line $u^{-}=u_{0}$ or $u^{-}=u_{0}^{-}$according to $u_{0}>\tilde{u}$ or $u_{0}<\tilde{u}$. The line $u^{-}=u_{0}$ or $u^{-}=u_{0}^{-}$, however, is also an integral curve of equation (14). That is, the integral curves must end at the singular points.

Similarly, we can prove the entropy condition is satisfied if at point $\left(\xi_{\alpha}^{0}, \eta_{\alpha}^{0}\right)$, the inflection point $u$ of $F$ is less than $u_{0}$.

We can picture all the integral curves in Figure 5.5.

$3^{\circ}$. Take $u^{+}=u^{* *}$ such that $u^{*}<u^{* *}<u_{0}$ and let the corresponding point be denoted by $u^{* *^{-}}$. Given a point $\left(\xi^{0}, \eta^{0}\right)$ on $u^{-}=u^{* *^{-}}$such that

$$
\begin{gathered}
\left.\frac{d \eta}{d \xi}\right|_{\left(\xi^{0}, \eta^{0}\right)}=\left(\frac{g\left(u^{* *^{-}}\right)-g\left(u_{0}\right)}{u^{* *^{-}}-u_{0}}-\eta^{0}\right) /\left(\frac{f\left(u^{* *^{-}}\right)-f\left(u_{0}\right)}{u^{* *^{-}}-u_{0}}-\xi^{0}\right)<0, \\
\frac{f\left(u^{* *^{-}}\right)-f\left(u_{0}\right)}{u^{* *^{-}}-u_{0}}<\xi^{0},
\end{gathered}
$$

then we can solve equation (14) through the point $\left(\xi^{0}, \eta^{0}\right)$ to get an integral curve satisfying the entropy condition (10) which will exist until $u^{+}=u_{0}$ or $u^{-}=u_{0}$.

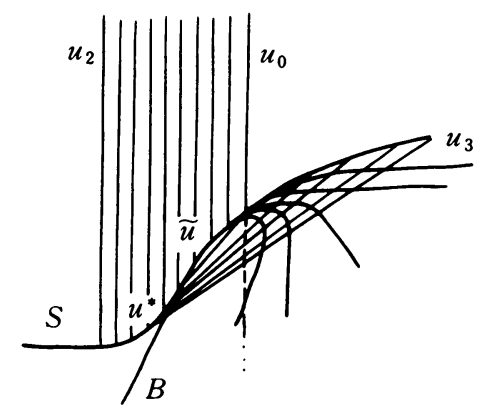

$u_{0} \geqq \widetilde{u}$

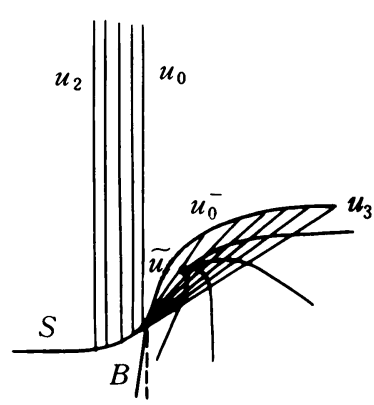

$u_{0}<\tilde{u}$

FIGURE 5.5. The integral curves to equation (14) 


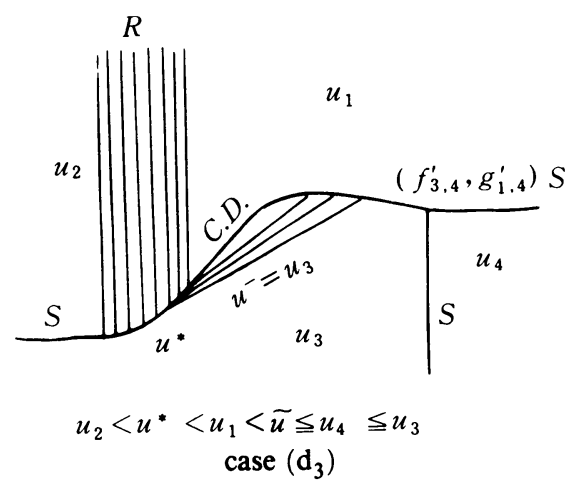

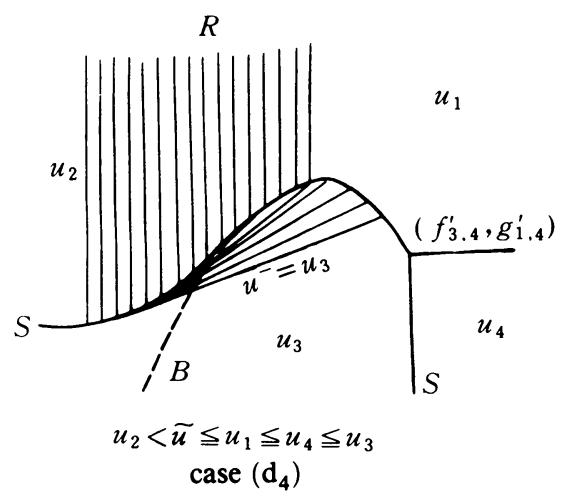

FIGURE 5.6

Now we can solve the remaining subcases of case (d).

$\left(\mathrm{d}_{3}\right): u_{2}<u^{*}<u_{1}<\tilde{u} \leq u_{4} \leq u_{3}$ and the point $\left(f_{3,4}^{\prime}, g_{1,4}^{\prime}\right)$ is below the line $u^{-}=u_{3}$. In this case, the shock connecting $\left(f_{1,3}^{\prime}, g_{1,3}^{\prime}\right)$ and $\left(f_{3,4}^{\prime}, g_{1,4}^{\prime}\right)$ penetrates the envelope rarefaction wave to arrive at the contact discontinuity line $u^{-}=u_{1}^{-}$(Figure 5.6, case $\left(\mathrm{d}_{3}\right)$ ).

$\left(\mathrm{d}_{4}\right): u_{2}<\tilde{u} \leq u_{1} \leq u_{4} \leq u_{3}$ and the point $\left(f_{4,3}^{\prime}, g_{1,4}^{\prime}\right)$ is below the line $u^{-}=u_{3}$. The shock connecting $\left(f_{3,4}^{\prime}, g_{1,4}^{\prime}\right)$ and $\left(f_{1,3}^{\prime}, g_{1,3}^{\prime}\right)$ penetrates the rarefaction wave to arrive at the point $\left(f^{\prime}\left(u_{1}\right), g^{\prime}\left(u_{1}\right)\right)$ on the base curve and thus vanishes (Figure 5.6, case $\left(\mathrm{d}_{4}\right)$ ).

$\left(\mathrm{d}_{5}\right): u_{2}<u^{*}<u_{1}<\tilde{u} \leq u_{4} \leq u_{3}$ and $\left(f_{3,4}^{\prime}, g_{1,4}^{\prime}\right)$ is above the line $u^{-}=u_{3}$ but below $u^{-}=u_{1}^{-}$. In this case, the shock $\xi=f_{3,4}^{\prime}$ should penetrate the envelope rarefaction wave to reach the point

$$
\left(f^{\prime}\left(u_{4}\right), g^{\prime}\left(u_{4}\right)\right) \text { or }\left(\frac{f\left(u_{4}^{-}\right)-f\left(u_{4}\right)}{u_{4}^{-}-u_{4}}, \frac{g\left(u_{4}^{-}\right)-g\left(u_{4}\right)}{u_{4}^{-}-u_{4}}\right) .
$$

However, it intersects $\eta=g_{1,4}^{\prime}$ at a point $A$ which satisfies the requirements of $3^{\circ}$. In fact,

$$
\xi_{A}>\frac{f\left(u_{A}^{-}\right)-f\left(u_{4}\right)}{u_{A}^{-}-u_{4}}>\frac{f\left(u_{A}^{-}\right)-f\left(u_{1}\right)}{u_{A}^{-}-u_{1}}
$$

$u_{3}>u_{A}^{-}>u_{4} \geq u_{1}, \eta=g_{1,4}^{\prime}<\left(g\left(u_{A}^{-}\right)-g\left(u_{1}\right)\right) /\left(u_{A}^{-}-u_{1}\right)$, therefore $d \eta /\left.d \xi\right|_{A}<$ 0 . Thus we continue the shock by the integral curve obtained in $3^{\circ}$ to arrive at

$$
\left(\frac{f\left(u_{1}\right)-f\left(u_{1}^{-}\right)}{u_{1}-u_{1}^{-}}, \frac{g\left(u_{1}\right)-g\left(u_{1}^{-}\right)}{u_{1}-u_{1}^{-}}\right)
$$

in this case or $\left(f^{\prime}\left(u_{1}\right), g^{\prime}\left(u_{1}\right)\right)$ in case $\left(\mathrm{d}_{6}\right)$ :

$\left(\mathrm{d}_{6}\right): u_{2}<\tilde{u} \leq u_{1} \leq u_{4} \leq u_{3}$ and $\left(f_{3,4}^{\prime}, g_{1,4}^{\prime}\right)$ is above the line $u^{-}=u_{3}$ (Figure 5.7). 


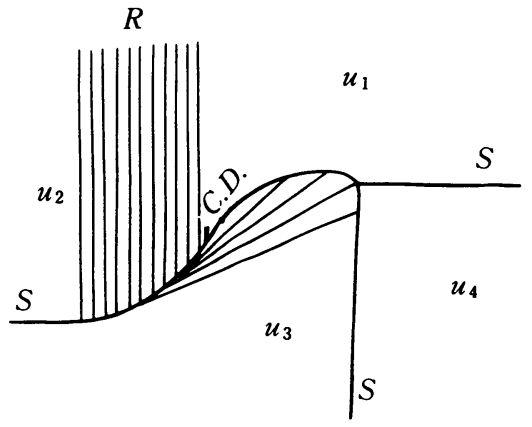

$u_{2}<u^{*}<u_{1}<\tilde{u} \leqq u_{4} \leqq u_{3}$ case $\left(d_{5}\right)$

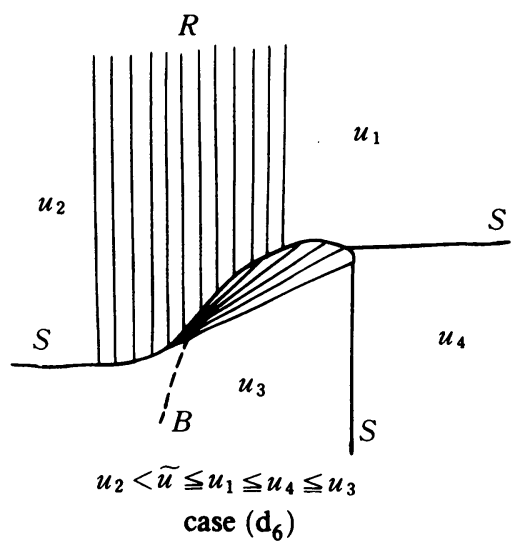

Figure 5.7

We next prove that point $A$ cannot be above the line $u^{-}=u_{1}^{-}$. In fact, we may assume $u_{1}<\tilde{u}, u_{4}<u_{1}^{-}$, since the other cases are obvious. The shock $\xi=f_{3,4}^{\prime}$ penetrates upward, and when it arrives at $u^{-}=u_{1}^{-}$, its tangent line must pass through the point

$$
\left(\frac{f\left(u_{1}^{-}\right)-f\left(u_{4}\right)}{u_{1}^{-}-u_{4}}, \frac{g\left(u_{1}^{-}\right)-g\left(u_{4}\right)}{u_{1}^{-}-u_{4}}\right)
$$

which is above the line $u^{-}=u_{1}^{-}$in view of inequality (16). But the penetrating line from $u^{-}=u_{3}$ to $u^{-}=u_{1}^{-}$is on the right of the straight segment connecting $\left(f_{4,1^{-}}^{\prime}, g_{4,1^{-}}^{\prime}\right)$ and the intersection point of $\xi=f_{3,4}^{\prime}$ with $u^{-}=u_{3}$. Noting that $f_{4,1^{-}}^{\prime}>f_{1,1^{-}}^{\prime}$, and $g_{1,4}^{\prime}<g_{1,1^{-}}^{\prime}$, we conclude that point $A$ is in the region

$$
\left\{(\xi, \eta) \mid \xi>f_{1,1^{-}}^{\prime}, \eta<g_{1,1^{-}}^{\prime}\right\} \text {. }
$$

We denote $\left(f\left(u_{1}^{-}\right)-f\left(u_{1}\right)\right) /\left(u_{1}^{-}-u_{1}\right)$ by $f_{1,1^{-}}^{\prime}$, and the result follows (Figure 5.8).

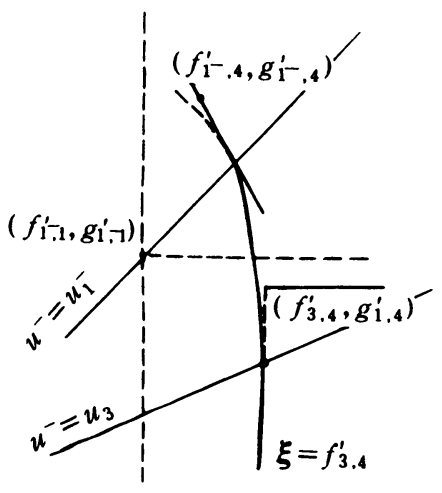

FigURE 5.8 
We have constructed completely all solutions to case (d), each of which satisfies the entropy condition.

\section{CASE (e): EXACTLY TWO RAREFACTION WAVES AND TWO SHOCKS}

We divide case (e) into two subcases, case $\left(e_{1}\right)$ and case $\left(e_{2}\right)$. Shocks in case $\left(e_{1}\right)$ are not neighbours to each other. There are also two possible cases in this subcase:

Case $\left(\mathrm{e}_{1}\right)_{1}: u_{4}>u_{1} \geq u_{3}>u_{2}$. This case is simple. We picture it as in Figure 6.1 .

Case $\left(\mathrm{e}_{1}\right)_{2}: u_{4}>u_{3}>u_{1}>u_{2}$. We can picture the solutions in this case as in Figure 6.2 from the proof of case $(\mathrm{d}), 1^{\circ}$.

Shocks in case $\left(e_{2}\right)$ are neighbours to each other. There are four possible orderings of initial data in this case.

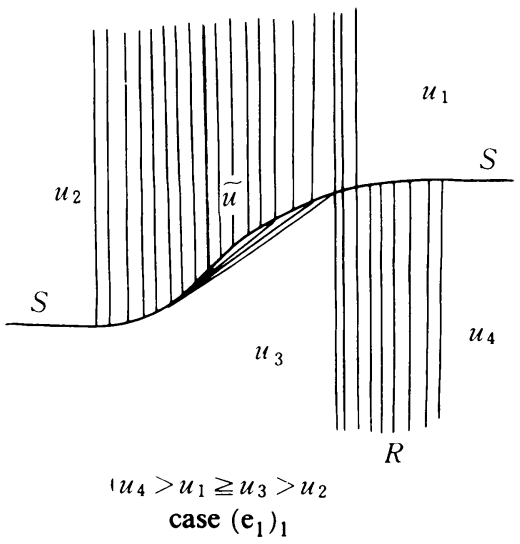

FIGURE 6.1

Case $\left(\mathrm{e}_{2}\right)_{1}: u_{4} \geq u_{2}>u_{1} \geq u_{3}$. We picture the solution as in Figure 6.3.

We know from case (c) that there exists a region in which the solution pieced together is over-determined. Thus from the intersection point $A$, we solve the following equation:

$$
\frac{d \eta}{d \xi}=\left(\frac{g\left(u^{-}\right)-g\left(u^{+}\right)}{u^{-}-u^{+}}-\eta\right) /\left(\frac{f\left(u^{-}\right)-f\left(u^{+}\right)}{u^{-}-u^{+}}-\xi\right), \quad \xi=f^{\prime}\left(u^{+}\right),
$$

where $u^{-}=u^{-}(\xi, \eta)$ satisfies

$$
\xi-\xi_{c}\left(u^{-}\right)=\frac{d \xi_{c}}{d \eta}\left(\eta-g^{\prime}\left(u^{++}\right)\right)
$$

and

$$
\left\{\begin{array}{c}
\frac{d \xi_{c}}{d \eta}=\frac{f_{++,-}^{\prime}-\xi_{c}\left(u^{-}\right)}{g_{++,-}^{\prime}-g^{\prime}\left(u^{++}\right)}=\frac{f^{\prime}\left(u^{-}\right)-f_{++,-}^{\prime}}{g^{\prime}\left(u^{-}\right)-g_{++,-}^{\prime}} \\
\eta=g^{\prime}\left(u^{++}\right) .
\end{array}\right.
$$

A smooth solution exists in a neighbourhood of point $A$ having one branch entering the region of the envelope rarefaction wave. $d \eta / d \xi$ remains finite until 


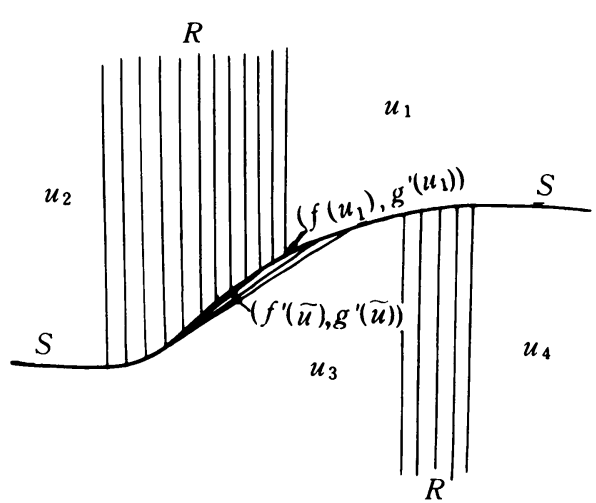

$u_{4}>u_{3}>u_{1} \geqq \tilde{u}>u_{2}$

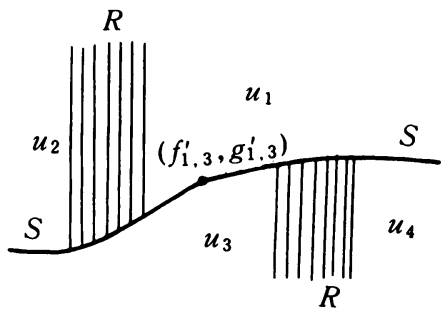

$u_{4}>u_{3}>u^{*} \geqq u_{1}>u_{2}$

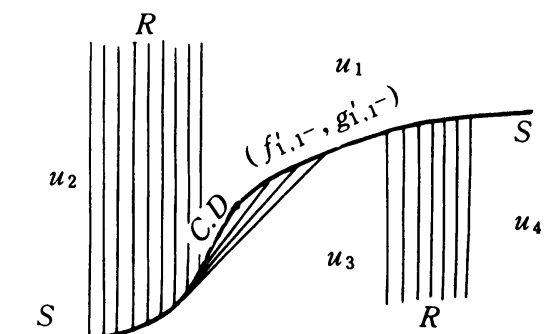

$$
u_{4}>u_{3}>\tilde{u}>u_{1}>u^{*}>u_{2}
$$

case $\left(e_{1}\right)_{2}$

\section{FIGURE 6.2}

$u^{+}=u^{-}$on the base curve, and the inflection point of $F(v)=(d \eta / d \xi) f(v)-$ $g(v)$ at point $A$ is larger than $u^{+}$at $A$. Thus the entropy condition (10) begins to be violated only when

$$
\frac{d \eta}{d \xi}=\frac{g_{+,-}^{\prime}-\eta}{f_{+,-}^{\prime}-f^{\prime}\left(u^{+}\right)}=\frac{g_{+,-}^{\prime}-g^{\prime}\left(u^{+}\right)}{f_{+,-}^{\prime}-f^{\prime}\left(u^{+}\right)}
$$

i.e., $\eta=g^{\prime}\left(u^{+}\right)$. Therefore, the solution will satisfy the entropy condition if it remains in the region of the envelope rarefaction wave. We claim that the solution does not intersect the base curve at a point $u^{+} \neq u^{-}$. Otherwise, there exists a $u^{+}>u^{-}$such that

$$
\frac{d \eta}{d \xi}=\frac{g_{+,-}^{\prime}-g^{\prime}\left(u^{+}\right)}{f_{+,-}^{\prime}-f^{\prime}\left(u^{+}\right)} \leq \frac{g^{\prime \prime}\left(u^{+}\right)}{f^{\prime \prime}\left(u^{+}\right)}
$$

and this is impossible. In order to prove that the solution does not intersect the line $u^{-}=u_{1}$ anymore, we suppose that at a point, it is tangent to a line $u^{-}=$ constant, then at the tangent point we have

$$
\frac{d \eta}{d \xi}=\frac{d \eta}{d \xi_{c}}=\frac{g_{+,-}^{\prime}-\eta}{f_{+,-}^{\prime}-f^{\prime}\left(u^{+}\right)}=\frac{g^{\prime}\left(u^{-}\right)-\eta}{f^{\prime}\left(u^{-}\right)-f^{\prime}\left(u^{+}\right)}=\frac{g^{\prime}\left(u^{-}\right)-g_{+,-}^{\prime}}{f^{\prime}\left(u^{-}\right)-f_{+,-}^{\prime}}
$$

This means that the solution is a half contact discontinuity at this point, and this is impossible except if $u^{-}=u^{+}$, due to (B). Thus the solution remains 


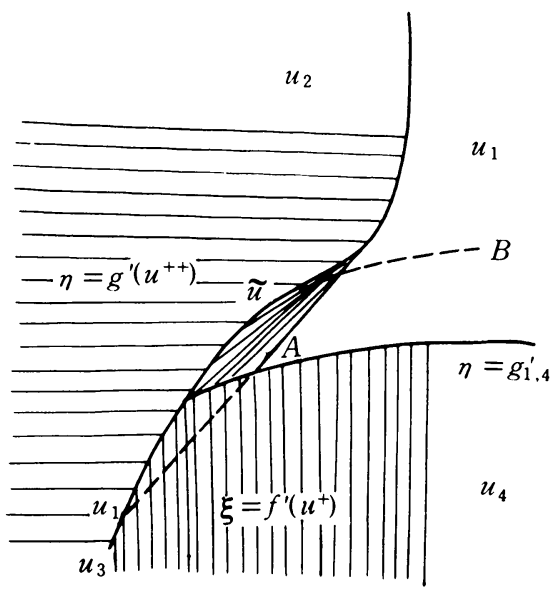

Figure 6.3

in the region of the envelope rarefaction wave until $u^{+}=u^{-}<\tilde{u}$ on the base curve. Now we can give Figure 6.3

Case $\left(\mathrm{e}_{2}\right)_{2}: u_{1} \geq u_{3}>u_{4} \geq u_{2}$. There are two possible cases in this case. The first case occurs when the shock $\xi=f_{3,4}^{\prime}$ meets $\eta=g^{\prime}\left(u_{4}\right)$ earlier than it meets the envelope rarefaction wave. The solution is similar to that in case $\left(e_{2}\right)_{1}$ (Figure 6.4). The second case occurs when the shock $\xi=f_{3,4}^{\prime}$ meets the lower boundary of the envelope rarefaction wave earlier than it meets $\eta=g^{\prime}\left(u_{4}\right)$. In this case, the shock $\xi=f_{3,4}^{\prime}$ first penetrates the envelope rarefaction wave. After intersecting $\eta=g^{\prime}\left(u_{4}\right)$, the shock continues in a way similar to that in case $\left(\mathrm{e}_{2}\right)_{1}$. Noting that $d \eta / d \xi<0$ before the solution intersects $\eta=g^{\prime}\left(u_{4}\right)$, we can prove analogously to case $\left(e_{2}\right)_{1}$ that the solution satisfies the entropy condition. We also note that the intersection point $A$ is always under the base curve. We can give the solution as in Figure 6.4, case $\left(e_{2}\right)_{2}$.

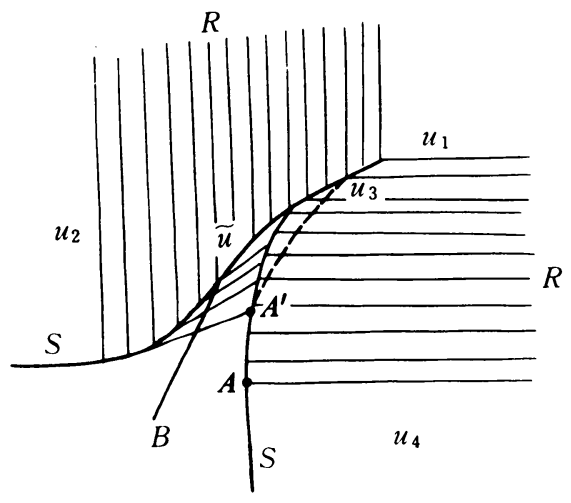

$\left(\mathrm{e}_{2}\right)_{1}: A^{\prime}$ higher than $A$

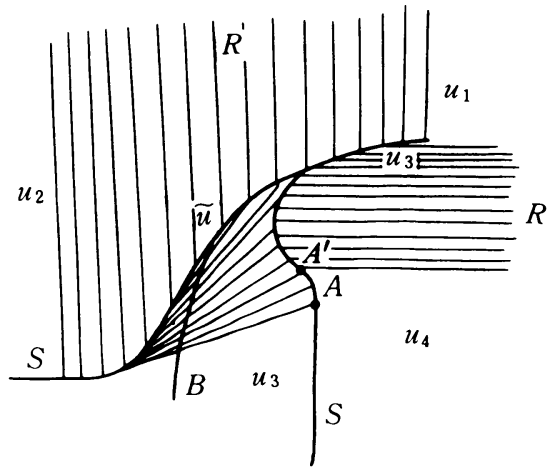

$\left(\mathrm{e}_{2}\right)_{2}: A^{\prime}$ lower than $A$

case $\left(e_{2}\right)_{1}$

Figure 6.4 


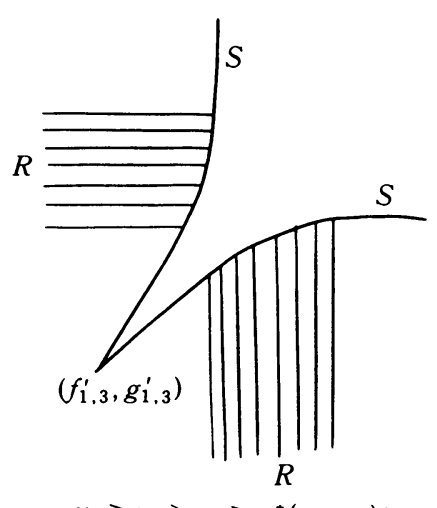

$u_{4} \geqq u_{2}>u_{3} \geqq u^{*}\left(u_{1}, u_{2}\right)>u_{1}$

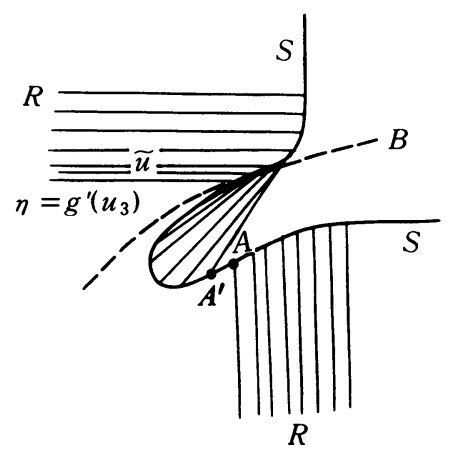

$u_{4} \geqq u_{2}>\widetilde{u} \geqq u_{3}>u_{1}$

with $A$ higher than $A^{\prime}$

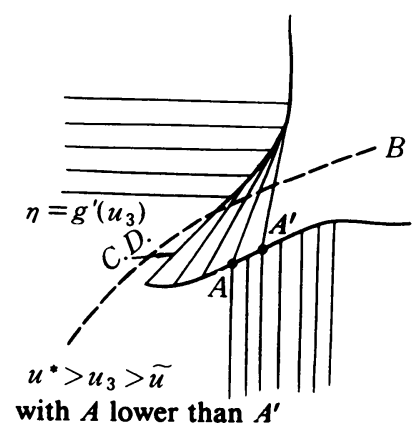

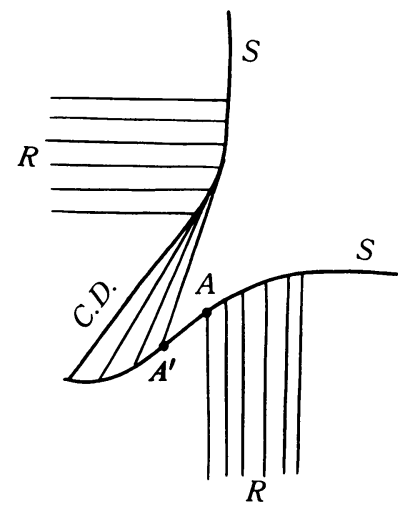

$u_{4} \geqq u_{2}>u^{*}>u_{3}>\tilde{u}>u_{1}$

with $A$ higher that $A^{\prime}$

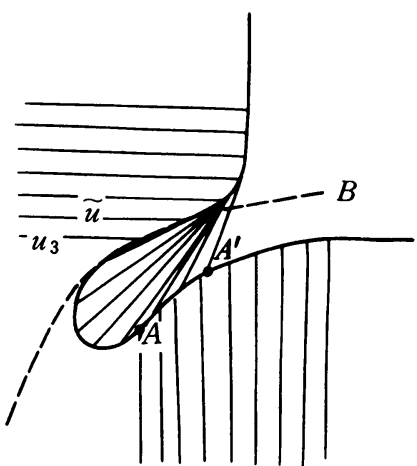

$u_{4} \geqq u_{2}>\widetilde{u} \geqq u_{3}>u_{1}$ with $A$ lower than $A^{\prime}$

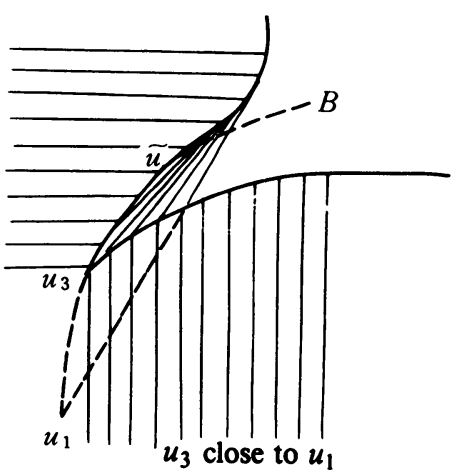

case $\left(e_{2}\right)_{3}$

FIGURE 6.5

Case $\left(\mathrm{e}_{2}\right)_{3}: u_{4} \geq u_{2}>u_{3}>u_{1}$. There are many subcases in this case. We picture them all in Figure 6.5. The proofs are easy, so we omit them.

Case $\left(\mathrm{e}_{2}\right)_{4}: u_{3}>u_{1}>u_{4} \geq u_{2}$. We only depict all cases (cf. Figure 6.6). 

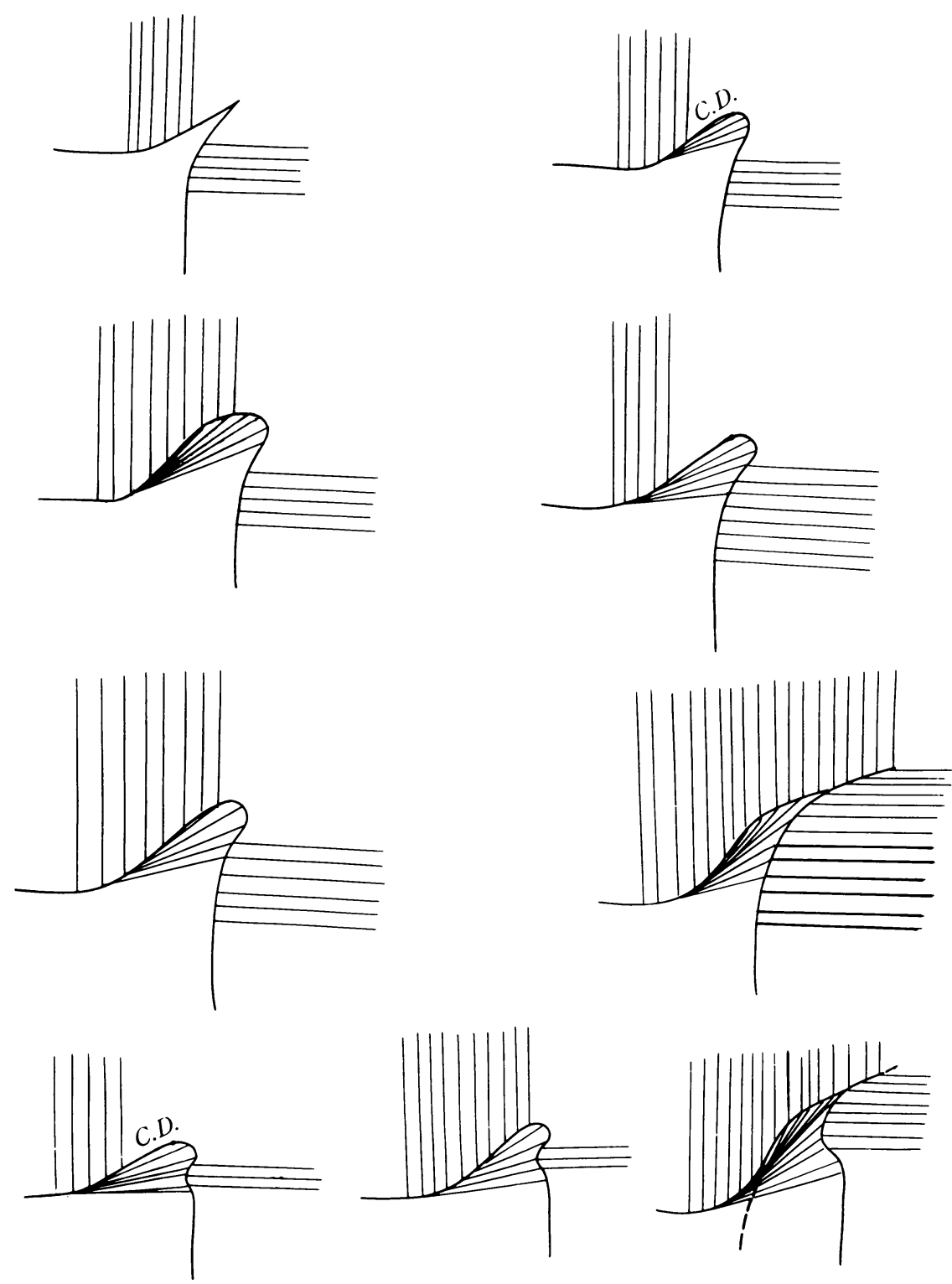

case $\left(e_{2}\right)_{4}$

FIGURE 6.6

\section{REFERENCES}

1. I. Gel' fand, Some problems in the theory of quasilinear equations, Uspekhi Mat. Nauk 14 (1959), 87-158; English transl., Amer. Math. Soc. Transl. (2) (1963), 295-381.

2. J. Smoller, Shock waves and reaction-diffusion equations, Springer-Verlag, 1983.

3. S. Kruzkov, First order quasilinear equations with several space variables, Mat. Sb. 123 (1970), 228-255; English transl., Math. USSR-Sb. 10 (1970), 217-243. 
4. D. Wagner, The Riemann problem in two space dimensions for a single conservation law, SIAM J. Math. Anal. 14 (1983), 534-559.

5. W. B. Lindquist, Construction of solutions to two-dimensional Riemann problem, and the scalar Riemann problem in one and two spatial dimensions: piecewise smooth solutions, New York Univ. Preprints, 1, 2, 1984.

6. J. Guckenheimer, Shocks and rarefactions in two space dimensions, Arch. Rational Mech. Anal. 59 (1975), 281-291.

7. Y. Val' ka, Discontinuous solutions of a multidimensional quasilinear equation (numerical experiments), U.S.S.R. Comput. Math. and Math. Phys. 8 (1968), 257-264.

8. Zhang Tong and Chen Gui-qiang, Some fundamental concepts about system of two spatial dimensional conservation laws, Acta Math. Sci. 6 (1986), 463-474.

Institute of Mathematics, Academia Sinica, Beijing, China

Current address (Yuxi Zheng): Department of Mathematics, University of California at Berkeley, Berkeley, California 94720 